\title{
RANKS OF ELLIPTIC CURVES OVER FUNCTION FIELDS
}

\author{
ALAN G.B. LAUDER
}

\begin{abstract}
We present experimental evidence to support the widely held belief that one half of all elliptic curves have infinitely many rational points. The method used to gather this evidence is a refinement of an algorithm due to the author which is based upon rigid and crystalline cohomology.
\end{abstract}

\section{Introduction}

There is a widely held belief that one half of all elliptic curves have infinitely many rational points, but all experimental data which has been collected so far suggests that the fraction is actually two thirds [2, Introduction, Items (1) and (2)]. The final purpose of this paper is to present experimental evidence supporting the widely held belief. Let us first digress, to explain the origin of the method which has been used to collect this evidence.

In an earlier paper, the present author introduced a new method for computing zeta functions of varieties over finite fields $[\mathbf{1 9}]$. The principal novelty of the method was that it proceeded by induction on the dimension of the variety. The author named it the fibration method. It was observed that the method appeared to be especially useful for the case of surfaces over finite fields which can be fibred into low genus curves. Given such a surface, the interesting part of its zeta function is exactly the L-function associated to the generic fibre - the generic fibre is a curve over a function field. According to a conjecture of Tate [26], the L-function reveals arithmetic properties of the curve, such as the rank of the group of rational points on its Jacobian. In the present paper, we carefully refine the fibration method, focussing ultimately on the case of elliptic curves over the rational function field, and use it to compute the L-functions of a randomly chosen sample of elliptic curves of extremely large conductor from a certain family. The computations reveal that around one half of those have rank, or at least analytic rank, greater than zero; see Section 9 .

Leaving aside this final motivating application, the central achievement of this paper is the further development of the fibration method, with an emphasis upon the computation of L-functions of hyperelliptic curves over the rational function field. We shall now sketch the improvements to the method which are obtained. We need to introduce some notation: For $q$ a power of a prime $p$, let $\mathbb{F}_{q}$ denote the finite field with $q$ elements of characteristic $p, K$ denote the unique unramified extension of the field of $p$-adic numbers of degree $\log _{p}(q)$, and $W$ denote the ring of integers of $K$.

The author is a Royal Society University Research Fellow.

Received 29 November 2007, revised 14 April 2008; published 30 July 2008.

2000 Mathematics Subject Classification 11G05, 11Y99, 14F30

(C) 2008, Alan G.B. Lauder 
The challenge which we address is that of efficiently computing the zeta function of a smooth variety over a finite field $\mathbb{F}_{q}$. Our methods are based upon rigid and crystalline cohomology. Using standard techniques, one sees that the problem of central interest is that of calculating the $p$ th power Frobenius action on the middledimensional rigid cohomology of the variety. This is a finite-dimensional vector space over $K$. In the case in which the original variety $V$ of dimension $n$ can be fibred into smooth varieties $V \rightarrow S$ over a curve $S \subset \mathbb{P}^{1}$, the paper [19] presents a method of reducing the problem of computing Frobenius on $H_{\text {rig }}^{n}(V)$ to that of computing Frobenius on $H_{\text {rig }}^{n-1}\left(V_{s}\right)$ for one smooth fibre $V_{s} \hookrightarrow V$ in the family. So the method proceeds by induction on the dimension. We now describe how the work in this paper refines the fibration method.

First, the method requires that a basis be found for the relative cohomology space $H_{\text {rig }}^{n-1}(V / S)$ such that the "matrix for the Gauss-Manin connection is Fuchsian" [19, Section 4]. This is an entirely reasonable assumption as such bases are known to exist for families, for example, with an irreducible monodromy group, at least after extending the base field; however, the problem of actually finding such bases is an interesting open one, related to Hilbert's 21st problem. An exposition of this problem is given, and modest contributions made, in Sections 7 and 8.3; however, this still remains an obstacle to the wider application of the fibration method.

Second, in [19, Section 9.3] some new ideas on how to significantly improve the practical performance of the fibration method are sketched, although no details are offered. We work out these ideas in great detail and use them in our calculations for elliptic curves. Specifically, let $X$ be some smooth compactification of the variety $V$. There is a finitely generated $W$-module $H_{c r i s}^{n}(X)$ attached to $X$ called the middledimensional crystalline cohomology, and a natural map $H_{c r i s}^{n}(X) \rightarrow H_{r i g}^{n}(V)$. The idea is that one computes a basis for the Frobenius-invariant $W$-lattice $H(X)_{\text {prim }}=$ $\operatorname{Im}\left(H_{c r i s}^{n}(X) \rightarrow H_{\text {rig }}^{n}(V)\right)$ and calculates Frobenius on this basis. Working with this basis, one can exploit the Hodge filtration to significantly reduce the $p$-adic precision to which the matrix for Frobenius needs to be computed. Rather than find $H(X)_{\text {prim }}$ itself, in Section 3 and 4 for certain surfaces $X$ we describe how to compute a lattice $H(X, k D)$ (where $D$ is a smooth divisor and $k$ a suitably large integer) which is very close, in a precise sense, to $H(X)_{\text {prim }}$, although possibly of larger rank. This lattice is sufficient for our application and easier to find.

Third, in [19, Conjecture 7.4] a conjecture is made on the vanishing of certain coefficients in the "relative Frobenius matrix" attached to families $V \rightarrow S$ of hyperelliptic curves and a choice of basis for $H_{r i g}^{1}(V / S)$. The truth of this conjecture immediately yields improvements to the running time of the original fibration method, see [19, Examples 9.2, 9.3], and also the refined method of this paper. We prove the conjecture, under modest hypotheses (Theorem 8.4). This also gives a practical improvement when one uses the deformation method to compute zeta functions of hyperelliptic curves over finite fields.

We now explain in greater detail the main theoretical contribution of this paper. Namely, our construction and application of $W$-lattices sitting in the middledimensional rigid cohomology of surfaces. This is the second refinement to the fibration method as introduced above.

For $U$ a smooth surface of degree $d$ in $\mathbb{A}_{\mathbb{F}_{q}}^{3}$, under certain assumptions for each $k \geqslant \max \{2 d-4,0\}$ we explicitly construct a full rank $W$-lattice $H(X, k D)$ sit- 
ting in the $K$-vector space $H_{\text {rig }}^{2}(U)$ (see the opening paragraph of Section 4). The motivation for constructing this lattice is Theorem 5.1. This theorem asserts that when $q=p$ is prime, if we compute an approximation $\tilde{A}$ to the ma$\operatorname{trix} A$ for the $p$ th power Frobenius map $F$ with respect to a basis for this lattice, the loss of precision in the calculation of $\operatorname{det}\left(1-p^{-1} \tilde{A} T\right)$ is bounded by $h^{2,0}+\left\lfloor\log _{p}(k+1)\right\rfloor$. Here $h^{2,0}$ is the geometric genus of a smooth compactification of a lifting to $W$ of $U$. Using an arbitrary basis of $H_{\text {rig }}^{2}(U)$, even when $\tilde{A}$ has entries in $W$ the author does not know how to improve the naive loss of precision bound of $\operatorname{dim}\left(H_{\text {rig }}^{2}(U)\right)-1$ (or $\left\lfloor\operatorname{dim}\left(H_{\text {rig }}^{2}(U)\right) / 2\right\rfloor-1$ using the functional equation). For the surfaces we implicitly consider in Section 9 , we have $p=7$ and $\left(h^{2,0}, \operatorname{dim}\left(H_{\text {rig }}^{2}(U)\right), d\right) \in\{(0,8,6),(1,20,12),(2,32,18),(3,44,24),(4,56,30)\}$. So the use of a basis for $H(X, k D)$ makes a very significant impact on precisions required. Lying behind Theorem 5.1 is the existence of an embedding $H(X, \log D) \rightarrow$ $H(X, k D)$ with cokernel killed by multiplication by $p^{\left\lfloor\log _{p}(k+1)\right\rfloor}$; here $H(X, \log D) \subset$ $H_{\text {rig }}^{2}(U)$ is a full rank $W$-lattice endowed with what we call a Frobenius-Hodge structure (Definition 3.12). In the case in which $U$ compactifies to a smooth surface $X$ in $\mathbb{P}_{\mathbb{F}_{q}}^{3}$ with smooth divisor at infinity, then our lattice $H(X, \log D)$ is exactly the log-crystalline cohomology of the pair $(X, D)$, where $D:=X \backslash U$ (Section 3). Unfortunately, for the surfaces we are mainly interested in (Sections 8 and 9), this compactification is not smooth. The surfaces $U$ we are mainly interested in do compactify to quasi-smooth surfaces $X$ in weighted projective spaces. We make the additional assumption that these weighted projective surfaces are quotients of smooth surfaces $\tilde{X}$ in $\mathbb{P}_{\mathbb{F}_{q}}^{3}$ with smooth divisor at infinity $\tilde{D}$ under a natural finite group action. The lattices $H(X, \log D)$ and $H(X, k D)$ are then defined by taking the invariant part under this group action of the lattices $H(\tilde{X}, \log \tilde{D})$ and $H(\tilde{X}, k \tilde{D})$ associated to the affine part $\tilde{U}:=\tilde{X} \backslash \tilde{D}$ of the smooth projective surface $\tilde{X}$ (Section 4.1). The lattices $H(X, \log D)$ and $H(X, k D)$ sit naturally in $H_{r i g}^{2}(U)$, and we prove that $H(X, \log D)$ has a Frobenius-Hodge structure (Section 4.2). However, we stress that we do not prove that $H(X, \log D)$ is the $\log$-crystalline cohomology of anything (although it undoubtedly is), see Note 4.8 .

The second and third refinements to the fibration method do not impact in any dramatic manner on the asymptotic analysis of the running time of the method; see Section 8.4 for a discussion. Their significance is perhaps best appreciated with reference to explicit examples. Regarding the third refinement, in [19, Example 9.1] a nine-fold speed-up is obtained by assuming the truth of [19, Conjecture 7.4]. Regarding the second refinement, in Section 9 the author computes, amongst other things, the L-functions of 1000 elliptic curves over $\mathbb{F}_{7}(t)$ which have discriminant a squarefree polynomial of degree 60 . For each curve, the computation took around 1 hour using the second and third refinements in this paper (the first is not relevant for the curves chosen). With only the second refinement, each computation would have taken around 4 hours. We note that in our computations in Section 9, and the timing presented in this paragraph, we use an algorithm due to K. Kedlaya for recovering Weil polynomials from their $p$-adic approximations [18, Section 4]. The output of our implementation of Kedlaya's algorithm is provably correct, although there are no explicit bounds on its running time. All of our calculations are done using the computer algebra system MAGMA.

We conclude the introduction with a brief mention of the contents of the other 
sections in this paper. Section 2 gives an overview of our refined fibration method and its relevance to the computation of L-functions. Section 6 proves some theorems of modest interest relating to one of the steps in the method, and includes a short discussion of the computation of the residue map.

The author has been assisted in many different ways by many different people, among them are: Noam Elkies, Victor Flynn, Ralf Gerkmann, Keith Gillow, Roger Heath-Brown, Hendrik Hubrechts, Nicholas Katz, Bernard Le Stum, Atsushi Shiho, William Stein, Balazs Szendroi, George Walker and Mark Watkins. He would like to thank them all. Especial thanks are due to Jan Denef, Kiran Kedlaya and Nobuo Tsuzuki, who answered with great patience many questions from the author. Finally, a great debt of gratitude is owed to A.J. de Jong, Bjorn Poonen and the anonymous referee.

\section{The refined fibration method}

The purpose of this section is to give an outline of the fibration method. The outline is based upon the original method presented in [19], incorporating refinements to the improvements crudely sketched in [19, Section 9.3]. To deal with the method in full would require the introduction of a great burden of notation, so we shall focus on aspects of it that are developed in this paper, and refer to [19] for details on parts which are fully investigated in that paper. We warn the reader that the notation for the different varieties required in this paper is not consistent with that in $[\mathbf{1 9}]$. The reason for this is that the focus of the two papers is quite different, and the notation from [19] would become burdensome in the present paper.

\section{1. $\quad$-adic rings and finite fields}

Let us first fix some notation which will be used throughout the paper. Let $\mathbb{F}_{q}$ be the finite field with $q$ elements of characteristic $p, W:=W\left(\mathbb{F}_{q}\right)$ be the ring of Witt vectors of $\mathbb{F}_{q}$, and $K$ be the fraction field of $W$. Thus $K$ is the unramified extension of the field of $p$-adic numbers of degree $\log _{p}(q)$, and $W$ is the ring of integers of $K$. Let $\sigma: W \rightarrow W$ and $\sigma_{K}: K \rightarrow K$ be the Frobenius automorphisms on $W$ and $K$ respectively, that is, the maps induced by functoriality by the $p$ th power map on $\mathbb{F}_{q}$. Let $\overline{\mathbb{F}}_{q}$ denote an algebraic closure of $\mathbb{F}_{q}$, and for each integer $k \geqslant 1$, let $\mathbb{F}_{q^{k}}$ denote the unique extension of degree $k$ of $\mathbb{F}_{q}$ in $\overline{\mathbb{F}}_{q}$.

\subsection{The zeta function and reduction to the middle dimension}

Let $X$ be a smooth projective variety of dimension $n$ over the finite field $\mathbb{F}_{q}$ of characteristic $p$. The zeta function $Z(X, T)$ is the formal power series

$$
Z(X, T):=\exp \left(\sum_{k=1}^{\infty}\left|X\left(\mathbb{F}_{q^{k}}\right)\right| \frac{T^{k}}{k}\right)
$$

which encodes the number of $\mathbb{F}_{q^{k}}$-rational points $\left|X\left(\mathbb{F}_{q^{k}}\right)\right|$ on $X$ over the different finite extensions $\mathbb{F}_{q^{k}}$ of the base field $\mathbb{F}_{q}$. By a famous theorem of Dwork, $Z(X, T)$ is a rational function. There is a formula from rigid cohomology [21, Pages 7-9]

$$
Z(X, T)=\prod_{i=0}^{2 n} \operatorname{det}\left(1-T q^{n} F_{q}^{-1} \mid H_{r i g}^{i}(X)\right)^{(-1)^{i+1}}
$$


here each $H_{r i g}^{i}(X)$ is a finite dimensional $K$-vector space, and $F_{q}$ a linear map induced by the $q$ th power map on the structure sheaf $\mathcal{O}_{X}$ of $X$. There is a factorisation $F_{q}=F^{\log _{p}(q)}$ where $F$ is the $\sigma_{K}$-linear map on $H_{\text {rig }}^{i}(X)$ induced by the $p$ th power map on $\mathcal{O}_{X}$.

The problem we consider is to compute $Z(X, T)$ given equations defining the variety $X$. We shall now try to convince the reader that the central problem is computing the factor with $i=n$.

First, we observe that if $X$ is a hypersurface in a space whose zeta function is known, then by Poincaré duality and the Lefschetz hyperplane theorem, one can reduce immediately to the case $i=n$. So let us suppose we are not in such a fortunate situation. We shall proceed by induction on the dimension, and assume one can compute zeta functions of smooth zero dimensional varieties - we offer no suggestions on how this base case should be tackled. By Poincaré duality, one need only calculate the factors in the formula for $0 \leqslant i \leqslant n$. Let us assume that $X \subseteq \mathbb{P}^{m}$, and there exists a smooth hyperplane section $Y \hookrightarrow X$ defined over the finite field $\mathbb{F}_{q}$ - this is certainly the case after extending the base field. The Lefschetz hyperplane theorem then reveals that the factors for $0 \leqslant i<n-1$ in the zeta function of $Z(Y, T)$ determine the corresponding factors in $Z(X, T)$. So by induction we reduce to the cases $i=n-1, n$. We suggest two different approaches to dealing with the case $i=n-1$. First, Deligne's pgcd theorem [7, Théorème 4.5.1] tells us that if one could compute the zeta functions of all smooth hyperplane sections in some Lefschetz pencil on $X$, then the $(n-1)$ st factor could be recovered by computing a greatest common divisor. Unfortunately, the author is not aware of any effective bounds on how many hyperplane sections need to be considered until one recovers the correct polynomial; in practice, though, this would seem a useful approach. Second, a more sophisticated, but rigorous, method for obtaining the $(n-1)$ st factor would be to use the spectral sequence in the fibration method, cf. the proof of Proposition 6.1. This should not be too difficult, although the author does not offer any details. In any case, we hope the reader is convinced that the case $i=n$ is the one of main interest.

The problem on which we now focus is that of calculating a $p$-adic approximation to a matrix for $F$ on the space $H_{r i g}^{n}(X)$.

\subsection{How the fibration method calculates in the middle dimension}

Let $\mathcal{X} \rightarrow \operatorname{Spec}(W)$ be a smooth and proper morphism of relative dimension $n$, $\mathcal{D}$ a divisor on $\mathcal{X}$ such that the restriction $\mathcal{D} \rightarrow \operatorname{Spec}(W)$ is smooth and proper and the complement $\mathcal{U}:=\mathcal{X} \backslash \mathcal{D}$ is affine.

\subsubsection{A fibration}

Since $\mathcal{U}$ is affine, we may embed it in some affine space $\mathbb{A}_{W}^{m}, m \geqslant n+1$. Choose a fibration $\mathbb{A}_{W}^{m} \rightarrow \mathbb{A}_{W}^{1}$. Let $\mathcal{U} \rightarrow \mathbb{A}_{W}^{1}$ be the restriction to $\mathcal{U}$. Choose $\mathcal{V} \subseteq \mathcal{U}$ so that the restriction $\mathcal{V} \rightarrow \mathcal{S} \subseteq \mathbb{A}_{W}^{1}$ exactly cuts out the smooth fibres in the morphism $\mathcal{U} \rightarrow \mathbb{A}_{W}^{1}$, and define $\mathcal{Z}:=\mathcal{U} \backslash \mathcal{V}$. By varying the embedding we may assume $\mathcal{U} \rightarrow \mathbb{A}_{W}^{1}$ is generically smooth, and so $\mathcal{Z}$ is of codimension one. Let $\mathcal{V}_{s}$ for $s \in \mathcal{S}(W)$ be some smooth fibre in the family $\mathcal{V} \rightarrow \mathcal{S}$ - one may need to extend the base field $\mathbb{F}_{q}$ to find a smooth fibre. Let the special fibres and generic fibres of $\mathcal{X}, \mathcal{D}, \mathcal{U}, \mathcal{V}, \mathcal{Z}$ be denoted $X, D, U, V, Z$ and $\mathcal{X}_{K}, \mathcal{D}_{K}, \mathcal{U}_{K}, \mathcal{V}_{K}, \mathcal{Z}_{K}$, respectively. Note that $n$ is the dimension of the special fibre $X$. 


\subsection{2. $\quad F$-crystals and isocrystals}

Definition 2.1. An F-crystal is a finitely generated $W$-module $M$ with a linear map $F: \sigma^{*} M \rightarrow M$ which becomes an isomorphism after tensoring with $K$. An Fisocrystal is a finite dimensional $K$-vector space $V$ with a linear map $F: \sigma_{K}^{*} V \rightarrow V$ which is an isomorphism.

That is, an $F$-crystal is a finitely generated $W$-module $M$ with a $\sigma$-linear map $F: M \rightarrow M$ which becomes an injection after tensoring by $K$, and an $F$-isocrystal is a finite dimensional $K$-vector space with an injective $\sigma_{K}$-linear map $F: V \rightarrow V$. Define the $F$-crystal $W(m)(m \in \mathbb{Z}, m \leqslant 0)$ to be the rank one module over $W$ where $F$ acts on the basis elements by multiplication by $p^{-m}$, and likewise for $K(m)(m \in \mathbb{Z})$. We define Tate twists $M(m):=M \otimes_{W} W(m)$ of an $F$-crystal $M$, and likewise for $F$-isocrystals.

Let $i$ be an integer. Associated to $X$ and $D$ are $F$-crystals

$$
H_{\text {cris }}^{i}(X), H_{\text {cris }}^{i}(D) \text {, and } H_{\text {cris }}^{i}((X, D))
$$

called the crystalline cohomology of $X$ and $D$ and log-crystalline cohomology of the pair $(X, D)$, respectively. The groups above fit together in an $F$-equivariant long exact sequence (see [1, Definition 2.3.3, Proposition 2.4.1, Proposition 2.2.8] and the references therein)

$$
\cdots \rightarrow H_{\text {cris }}^{i}(X) \rightarrow H_{\text {cris }}^{i}((X, D)) \rightarrow H_{\text {cris }}^{i-1}(D)(-1) \rightarrow \cdots .
$$

Associated to $X, D, U, V, Z$ and $V_{s}$ are $F$-isocrystals

$$
H_{\text {rig }}^{i}(X), H_{\text {rig }}^{i}(D), H_{\text {rig }}^{i}(U), H_{\text {rig }}^{i}(V) \text {, and } H_{\text {rig }}^{i}\left(V_{s}\right)
$$

called the rigid cohomology, and $F$-isocrystals $H_{r i g, Z}^{i}(U)$ called the rigid cohomology of $U$ with support in $Z$. There is an $F$-equivariant long exact sequence [17, Section 4.4]

$$
\cdots \rightarrow H_{r i g, Z}^{i}(U) \rightarrow H_{r i g}^{i}(U) \rightarrow H_{r i g}^{i}(V) \rightarrow \cdots
$$

There are natural maps

$$
\begin{aligned}
H_{\text {cris }}^{i}(X) & \rightarrow H_{r i g}^{i}(X) \\
H_{\text {cris }}^{i}((X, D)) & \rightarrow H_{\text {rig }}^{i}(U)
\end{aligned}
$$

which are injections modulo torsion with images full rank $W$-lattices [1, Definition 2.3.3, Proposition 2.4.1]. We shall use the same symbol $F$ to denote the Frobenius action on each of the $F$-crystals and $F$-isocrystals listed above.

Definition 2.2. The following images are Frobenius-invariant $W$-lattices sitting naturally in $K$-vector spaces; that is, F-crystals embedded naturally in F-isocrystals.

$$
\begin{array}{ll}
H(X)_{\text {prim }} & :=\operatorname{Im}\left(H_{\text {cris }}^{n}(X) \rightarrow H_{\text {rig }}^{n}(U)\right) \\
H(X, \log D) & :=\operatorname{Im}\left(H_{\text {cris }}^{n}((X, D)) \rightarrow H_{\text {rig }}^{n}(U)\right) \\
H(X) & :=\operatorname{Im}\left(H_{\text {cris }}^{n}(X) \rightarrow H_{\text {rig }}^{n}(X)\right) .
\end{array}
$$

For the smooth surfaces in $\mathbb{P}_{\mathbb{F}_{q}}^{3}$ we consider in Section 3, the lattice $H(X)_{\text {prim }}$ is exactly "primitive" middle-dimensional crystalline cohomology. 


\subsubsection{A summary of the method}

We wish to calculate a $p$-adic approximation to a matrix for the action of $F$ on the full rank Frobenius-invariant $W$-lattice $H(X)$. The reason why it is important to work with a basis for this lattice rather than an arbitrary basis is given in [19, Section 9.3.2]. Essentially, one can exploit the Hodge filtration to significantly reduce the power of $p$ to which the matrix needs to be calculated. We first note that it is both easier and more efficient to compute a matrix for $F$ on the $W$ lattice $H(X)_{\text {prim. }}$. It is easier because $U$ is affine and so the elements in $H_{\text {rig }}^{n}(U)$ can be described via $n$-forms defined on $\mathcal{U}_{K}$. It is more efficient, at least for the case $n=2$ of surfaces, since $H(X)_{\text {prim }}$ is the quotient of $H(X)$ by the rank one lattice generated by the class of the curve $D$, and the Frobenius action on the class of this curve is just multiplication by $q$.

The steps in our algorithm for calculating $F$ on the lattice $H(X)_{\text {prim }}$ and our work upon the different steps can be tersely summarised as follows.

- SteP 1: Find a set of $n$-forms in $\Gamma\left(\mathcal{U}_{K}, \Omega_{\mathcal{U}_{K}}^{n}\right)$ whose image in $H_{\text {rig }}^{n}(U)$ is a basis for the full rank $W$-lattice $H(X, \log D)$. [This is the problem of finding explicit bases in log-crystalline cohomology. It is solved for certain surfaces in weighted projective spaces in Sections 3 and 4.]

- Step 2: Use the long exact sequence for crystalline and log-crystalline cohomology (1) to compute a basis for the sublattice $H(X)_{\text {prim }} \subseteq H(X, \log D)$ from one for $H(X, \log D)$ itself. [This is the problem of explicitly computing the residue map. It is briefly discussed in Section 6.3.]

- Step 3: Use the long exact sequence in rigid cohomology (2) to map the basis elements for $H(X)_{\text {prim }}$ into the space $H_{\text {rig }}^{n}(V)$. Thus one reduces the problem to that of calculating the action of $F$ on the space $H_{r i g}^{n}(V)$. [We discuss this in Section 6 for certain surfaces in weighted projective spaces.]

- Step 4: Use the smooth fibration $V \rightarrow S$ to reduce the problem to that of calculating the action of $F$ on the space $H_{r i g}^{n-1}\left(V_{s}\right)$ for one smooth fibre $V_{s} \hookrightarrow V$ defined over $\mathbb{F}_{q}$. [This is the heart of the fibration method, and is dealt with in detail in $[\mathbf{1 9}$, Sections $3,4,5]$. One outstanding problem which is not fully resolved in $[\mathbf{1 9}]$ is that of finding a Fuchsian basis. This problem is explained in Section 7, and in Section 8.3 a solution for elliptic curves is given using an algorithm due to W. Dekkers. Unfortunately, our solution may require one to extend the base field.]

In practice, it is not always necessary or desirable to carry out Steps 1 and 2 exactly as stated. First, it is easier though perhaps slightly less efficient to compute a matrix for $F$ on the full $W$-lattice $H(X, \log D)$ rather than its sublattice $H(X)_{\text {prim }}$; that is, to omit Step 2. Second, it is not necessary to find $H(X, \log D)$ exactly, as a lattice which is very close to it will be sufficient. For example, in our application to surfaces $X$ we actually work with a lattice $H(X, k D)(k \gg 0$, see Theorem 3.11 and Definition 4.9) such that

$$
H(X)_{\text {prim }} \stackrel{i}{\hookrightarrow} H(X, \log D) \stackrel{\rho}{\hookrightarrow} H(X, k D) \subseteq H_{r i g}^{2}(U)
$$

where the cokernel of the embedding $\rho$ is killed by $p^{\left\lfloor\log _{p}(k+1)\right\rfloor}$. For certain elliptic surfaces the map $i$ is an isomorphism and when $p$ is large enough relative to the degree of the surface so is $\rho$, so we find $H(X)_{\text {prim }}$ exactly. For the examples we 
present in Section 9, the corank of the image of $i$ is 2, and the map $\rho$ has cokernel killed by at worst $p^{2}$. So $H(X, k D)$ contains a sublattice of corank 2 which is very close to $H(X)_{\text {prim }}$. This is good enough for our application. Theorem 5.1 exactly quantifies the benefits one obtains from working with a basis of the lattice $H(X, k D)$.

\subsection{L-functions}

We now explain the relevance of our algorithm to the computation of L-functions of certain curves. For $X$ a smooth surface in $\mathbb{P}_{\mathbb{F}_{q}}^{3}$ construct a Lefschetz pencil $\hat{X} \rightarrow$ $\mathbb{P}_{\mathbb{F}_{q}}^{1}$ on $X$ with axis lying on the divisor at infinity (possibly after extending the base field), see [23, Pages 197-199]. Choose a section $\alpha: \mathbb{P}_{\mathbb{F}_{q}}^{1} \rightarrow \hat{X}$; so $\alpha\left(\mathbb{P}_{\mathbb{F}_{q}}^{1}\right)$ is the blow-up of one of the points on the intersection of $X$ with the axis of the pencil. Let $\hat{X}_{\eta}$ denote the generic curve of the family $\hat{X} \rightarrow \mathbb{P}_{\mathbb{F}_{q}}^{1}$, and $U$ be the affine piece of $X$. Then

$$
H(X)_{\text {prim }}=\operatorname{Image}\left(H_{\text {cris }}^{2}(\hat{X}) \rightarrow H_{\text {rig }}^{2}(U)\right),
$$

where $H(X)_{\text {prim }}$ is the lattice considered in Section 2.3.3. We shall define the Lfunction of the generic curve $\hat{X}_{\eta}$ in the family $\hat{X} \rightarrow \mathbb{P}_{\mathbb{F}_{q}}^{1}$ to be

$$
L\left(\hat{X}_{\eta}, T\right):=\operatorname{det}\left(1-T F_{q} \mid H(X)_{\text {prim }} \otimes_{W} K\right) .
$$

This agrees with the usual definition, since it is exactly the "interesting part" of the zeta function of $\hat{X}$, obtained from $P_{2}(\hat{X}, T):=\operatorname{det}\left(1-T F_{q} \mid H_{\text {rig }}^{2}(\hat{X})\right)$ by removing factors coming from the classes of $\alpha\left(\mathbb{P}_{\mathbb{F}_{q}}^{1}\right)$ and a smooth fibre in $H_{\text {cris }}^{2}(\hat{X})$.

In our application to elliptic curves in Weierstrass form, we proceed in a more circuitous manner since the associated affine surface $U$ does not compactify to a smooth surface in $\mathbb{P}_{\mathbb{F}_{q}}^{3}$. However, under certain assumptions we are still able to define a lattice $H(X)_{\text {prim }} \subset H_{\text {rig }}^{2}(U)$ such that $\operatorname{det}\left(1-T F_{q} \mid H(X)_{\text {prim }} \otimes_{W} K\right)$ is the "interesting part" of the zeta function of $U$ and the L-function of the elliptic curve (Section 4.2).

\section{Crystalline cohomology of smooth surfaces in $\mathbb{P}^{3}$}

In this section we address the problem of finding the lattice $H(X, \log D)=$ $\operatorname{Im}\left(H_{c r i s}^{n}((X, D)) \rightarrow H_{\text {rig }}^{n}(U)\right)$, in the case in which $X$ is a smooth surface of degree $d$ sitting in projective space $\mathbb{P}_{\mathbb{F}_{q}}^{3}$. We shall proceed in some generality in Section 3.1 , before specialising to smooth surfaces in $\mathbb{P}_{\mathbb{F}_{q}}^{3}$ in Sections 3.2 and 3.3. Ultimately, we will be satisfied with finding a lattice $H(X, k D)$, for any $k \geqslant \max \{2 d-4,0\}$, which contains $H(X, \log D)$ as a sublattice, with the quotient an abelian group killed by multiplication by $p^{\left\lfloor\log _{p}(k+1)\right\rfloor}$. We use these lattices to define analogous lattices in Section 4 associated to certain weighted projective surfaces, and explain why all of these lattices are useful in Section 5.

\subsection{Some general results for surfaces}

Let $\mathcal{X} \rightarrow \operatorname{Spec}(W)$ be a smooth and proper morphism of relative dimension $2, \mathcal{D}$ a divisor on $\mathcal{X}$ such that the restriction $\mathcal{D} \rightarrow \operatorname{Spec}(W)$ is smooth and proper and the complement $\mathcal{U}:=\mathcal{X} \backslash \mathcal{D}$ is affine. Let $X, D, U$ (respectively $\mathcal{X}_{K}, \mathcal{D}_{K}, \mathcal{U}_{K}$ ) denote the special (respectively generic) fibres of $\mathcal{X}, \mathcal{D}$ and $\mathcal{U}$. 
3.1.1. Crystalline and de Rham cohomology

For $i \geqslant 0$, denote by $\Omega_{\mathcal{X}}^{i}:=\wedge^{i} \Omega_{\mathcal{X}}^{1}$ the sheaf of differential $i$-forms on $\mathcal{X}$, and by

$$
\Omega_{\mathcal{X}}^{\bullet}: 0 \rightarrow \mathcal{O}_{\mathcal{X}} \rightarrow \Omega_{\mathcal{X}} \rightarrow \Omega_{\mathcal{X}}^{2} \rightarrow 0
$$

the algebraic de Rham complex of $\mathcal{X}$. The algebraic de Rham cohomology $H_{d R}(\mathcal{X})$ of $\mathcal{X}$ is by definition the hypercohomology $\mathbb{H}\left(\Omega_{\mathcal{X}}^{\bullet}\right)$ of this complex. This is a finitely generated $W$-module. The complex $\Omega_{\mathcal{D}}^{\bullet}$ and finitely generated $W$-module $H_{d R}(\mathcal{D}):=$ $\mathbb{H}\left(\Omega_{\mathcal{D}}^{\bullet}\right)$ are defined in an analogous manner. For $i \geqslant 0$, denote by $\Omega^{i}(\log \mathcal{D}):=$ $\wedge^{i} \Omega_{\mathcal{X}}^{1}(\log \mathcal{D})$ the sheaf of differential $i$-forms on $X$ with logarithmic poles along $\mathcal{D}$, and by

$$
\Omega_{\mathcal{X}}^{\bullet}(\log \mathcal{D}): 0 \rightarrow \mathcal{O}_{\mathcal{X}} \rightarrow \Omega_{\mathcal{X}}(\log \mathcal{D}) \rightarrow \Omega_{\mathcal{X}}^{2}(\log \mathcal{D}) \rightarrow 0
$$

the logarithmic de Rham complex [1, Definition 2.2.2]. The log-de Rham cohomology $H_{d R}((\mathcal{X}, \mathcal{D}))$ is by definition the hypercohomology $\mathbb{H}\left(\Omega_{\mathcal{X}}^{\bullet}(\log \mathcal{D})\right)$ of this complex. This is a finitely generated $W$-module. There is an exact sequence

$$
0 \rightarrow \Omega_{\mathcal{X}}^{\bullet} \rightarrow \Omega_{\mathcal{X}}^{\bullet}(\log \mathcal{D}) \stackrel{\text { Res }}{\rightarrow} j_{*} \Omega_{\mathcal{D}}^{\bullet}[+1] \rightarrow 0
$$

where the map Res is the residue map and $j: \mathcal{D} \hookrightarrow \mathcal{X}$ [1, Proposition 2.2.8]. This induces a long exact sequence on the hypercohomology groups:

$$
\cdots \rightarrow H_{d R}^{i}(\mathcal{X}) \rightarrow H_{d R}^{i}((\mathcal{X}, \mathcal{D})) \rightarrow H_{d R}^{i-1}(\mathcal{D}) \rightarrow \cdots .
$$

Let $X$ and $D$ be the special fibres of $\mathcal{X}$ and $\mathcal{D}$ respectively. Associated to the smooth pair of $\mathbb{F}_{q}$-varieties $(X, D)$ are finitely generated $W$-modules with canonical isomorphisms [1, Definition 2.3.3, Proposition 2.4.1]

$$
H_{\text {cris }}(X) \cong H_{d R}(\mathcal{X}), H_{\text {cris }}(D) \cong H_{d R}(\mathcal{D}), H_{\text {cris }}((X, D)) \cong H_{d R}((\mathcal{X}, \mathcal{D})) .
$$

These are the crystalline and log-crystalline cohomology groups. The aim of this section is to give an explicit presentation of the log-crystalline cohomology groups $H_{\text {cris }}^{i}((X, D))$.

\subsubsection{The hypercohomology of the logarithmic de Rham complex}

For $i, k \geqslant 0$, let $\Omega^{i}(k \mathcal{D}):=\Omega_{\mathcal{X}}^{i} \otimes_{\mathcal{O}_{\mathcal{X}}} \mathcal{O}_{\mathcal{X}}(k \mathcal{D})$ denote the sheaf of differential $i$-forms on $\mathcal{X}$ with poles of order bounded by $k$ along $\mathcal{D}$. Consider the complex

$$
\Omega_{\mathcal{X}}((k+\bullet) \mathcal{D}): 0 \rightarrow \mathcal{O}_{\mathcal{X}}(k \mathcal{D}) \rightarrow \Omega_{\mathcal{X}}((k+1) \mathcal{D}) \rightarrow \Omega_{\mathcal{X}}^{2}((k+2) \mathcal{D}) \rightarrow 0 .
$$

THEOREM 3.1. The cokernels of the maps of homology sheaves induced by the natural map of complexes of sheaves

$$
\Omega_{\mathcal{X}}^{\bullet}(\log \mathcal{D}) \hookrightarrow \Omega_{\mathcal{X}}^{\bullet}((k+\bullet) \mathcal{D})
$$

are killed by multiplication by $\operatorname{lcm}\{1,2, \ldots, k+1\}$. Moreover, the induced maps on homology sheaves are injective.

Proof. We will show using étale local coordinates that for each $0 \leqslant i \leqslant 2$ the map of homology sheaves

$$
\mathcal{H}^{i}\left(\Omega_{\mathcal{X}}^{\bullet}(\log \mathcal{D})\right) \rightarrow \mathcal{H}^{i}\left(\Omega_{\mathcal{X}}^{\bullet}((k+\bullet) \mathcal{D})\right)
$$

is an injection and has cokernel killed by $\operatorname{lcm}\{1,2, \ldots, k+i-1\}$.

For points not lying on $\mathcal{D}$ there is nothing to prove, since locally at these points the sheaves are isomorphic. Etale locally around a point on $\mathcal{D}$ the pair $(\mathcal{X}, \mathcal{D})$ looks 
like the hyperplane section $x=0$ of an open $\operatorname{subset} \operatorname{Spec}(R)$ of $\operatorname{Spec}(W[x, y])$. By a further Zariski localisation we may assume $R$ contains a subring isomorphic to $R /(x)$, and derivation w.r.t. $x$ is trivial on this subring. (That is, with $S:=\left\{y_{0} \mid\left(0, y_{0}\right) \notin \operatorname{Spec}(R)\right\}$, replace $R$ by $R^{\prime}:=R\left[1 /\left(y-y_{0}\right) ; y_{0} \in S\right]$ and then $W[y]\left[1 /\left(y-y_{0}\right) ; y_{0} \in S\right]$ is the required subring of $R^{\prime}$ isomorphic to $R^{\prime} /(x)$; then replace the notation $R^{\prime}$ by $R$.)

We shall use subscripts $x$ and $y$ to denote partial differentiation w.r.t. $x$ and $y$, respectively. Let $\ell$ be a positive integer, and define $n_{\ell}:=\operatorname{lcm}\{1,2, \ldots, \ell-1\}$. For $a=$ $\sum_{i=-\ell}^{-1} a_{i}(y) x^{i} \in R\left[x^{-1}\right]$ where $a_{i}(y) \in R /(x) \subset R$, define $\hat{a}:=\sum_{i=-\ell}^{-2}\left(a_{i}(y) /(i+\right.$ 1)) $x^{i+1} \in R\left[x^{-1}\right] \otimes_{W} K$. Then $(\hat{a})_{x}=a+\left(a_{-1}(y) / x\right)$ and $n_{\ell} \hat{a} \in R\left[x^{-1}\right]$.

For $i=0$ there is nothing to prove since the closed 0 -forms are just the locally constant functions for both sheaves. For $i=2$, let $\alpha(x, y) d x \wedge d y \in \Omega_{R\left[x^{-1}\right]}^{2}$ be a closed 2 -form with a pole of order $k+2$. Let $\alpha=a(x, y)+b(x, y)$ where $b$ has no pole along $\mathcal{D}$, and write $a$ as in the preceding paragraph, taking $\ell=k+2$. Define $\beta:=\hat{a} d y \in \Omega_{R\left[x^{-1}\right]}^{1} \otimes_{W} K$. Then $\alpha d x \wedge d y-d \beta=\frac{a_{-1} d x}{x} \wedge d y+\gamma$ where the 2-form $\gamma$ has no pole along $\mathcal{D}$. Moreover, $n_{k+2} \beta \in \Omega_{R\left[x^{-1}\right]}$. Thus after multiplication by $n_{k+2}$ locally any 2 -form can be written modulo exact 2 -forms as a 2 -form with a $\log$-pole along $\mathcal{D}$. This proves the claim on the cokernel for $i=2$.

We now consider the cokernel for $i=1$. Let $\alpha=\beta(x, y) d x+\gamma(x, y) d y \in \Omega_{R\left[x^{-1}\right]}^{1}$ be a closed 1-form with a pole of order $k+1$ along $\mathcal{D}$. Since $\alpha$ is closed, $\beta_{y}=-\gamma_{x}$. As before, write $\beta=a(x, y)+b(x, y)$ where $b$ has no pole along $\mathcal{D}$, and write $a$ as above, taking $\ell=k+1$. Then $\alpha-d(\hat{a})=\frac{a_{-1} d x}{x}+\left(\gamma-(\hat{a})_{y}\right) d y$. Using the equation $\beta_{y}=-\gamma_{x}$ we see that $\gamma-(\hat{a})_{y}$ has no pole along $\mathcal{D}$. Thus after multiplication by $n_{k+1}$ locally any closed 1 -form can be written modulo exact 1 -forms as a 1 -form with a log-pole along $\mathcal{D}$.

We now prove the maps on sheaves are injective by examining kernels. For $i=0$ there is nothing to prove. For $i=1$, a closed 1-form $\alpha \in \Omega_{R\left[x^{-1}\right]}^{1}$ with a log-pole has the form $\beta(x, y) \frac{d x}{x}+\gamma(x, y) d y$ where the functions $\beta, \gamma \in R$ have no poles along $\mathcal{D}$, with $(\beta / x)_{y}=-\gamma_{x}$. Suppose that $\alpha=d f$ for some function $f(x, y) \in R\left[x^{-1}\right]$ with a pole of order $\ell \geqslant 1$ along $\mathcal{D}$. Since $f_{y}=\gamma$, we see $f_{y}$ has no pole along $\mathcal{D}$, so $f$ must be independent of $y$ with $\gamma=f_{y}=0$. Thus $(\beta / x)_{y}=0$, so $\beta$ does not depend on $y$, and we have $f(x)_{x}=\beta(x) / x$. This can only be true if $\beta(x) / x$ has no pole along $\mathcal{D}$, and so neither does $f$, a contradiction. Thus no such $f$ can exist, and the map on homology sheaves is injective for $i=1$.

Now let $i=2$. A (closed) 2-form $\alpha \in \Omega_{R\left[x^{-1}\right]}^{2}$ with a log-pole locally has the form $\beta(x, y) \frac{d x}{x} \wedge d y$ for some function $\beta \in R$ with no pole along $\mathcal{D}$. Suppose that $\alpha=d \gamma$ where the 1 -form $\gamma \in \Omega_{R\left[x^{-1}\right]}^{1}$ has a pole of order $\ell \geqslant 1$ along $\mathcal{D}$. We shall show that the exists a 1 -form $\gamma^{\prime} \in \Omega_{R\left[x^{-1}\right]}^{1}$ with a log-pole along $\mathcal{D}$ such that $\alpha=d \gamma^{\prime}$. This proves our map of homology sheaves is injective on 2-forms.

Write $\gamma=a(x, y) d x+b(x, y) d y$. Let $a=a^{\prime \prime}+a^{\prime}$ and $b=b^{\prime \prime}+b^{\prime}$ where $a^{\prime}$ has a simple pole along $\mathcal{D}$ with $a^{\prime \prime}:=a-a^{\prime}=\sum_{i=-\ell}^{-2} a_{i}(y) x^{i}$, and $b^{\prime}$ has no pole along $\mathcal{D}$ with $b^{\prime \prime}:=b-b^{\prime}=\sum_{i=-\ell}^{-1} b_{i}(y) x^{i}$. Then since $\beta(x, y) / x=b_{x}-a_{y}$ has a log-pole, we see $b_{-\ell}=0$, and for $i=-\ell,-\ell+1, \ldots,-2$ we have $\left(a_{i}\right)_{y}=(i+1) b_{i+1}$. Hence $\gamma^{\prime \prime}:=a^{\prime \prime} d x+b^{\prime \prime} d y$ is a closed 1-form. Defining $\gamma^{\prime}:=a^{\prime} d x+b^{\prime} d y$ one sees that $\gamma^{\prime}$ has a log-pole along $\mathcal{D}$. Moreover, $\alpha=d \gamma=d\left(\gamma^{\prime}+\gamma^{\prime \prime}\right)=d \gamma^{\prime}$, as required. 
Note 3.2. An analogue of the claim on the cokernel is proved in [1, Theorem 2.2.5] with the complex $\Omega_{\mathcal{X}}((k+\bullet) \mathcal{D})$ replaced by the twisted logarithmic complex whose $i$ th term is $\Omega_{\mathcal{X}}^{i}(\log \mathcal{D}) \otimes_{\mathcal{O}_{\mathcal{X}}} \mathcal{O}_{\mathcal{X}}(k \mathcal{D})$, for the more general situation in which $\mathcal{D}$ is a smooth normal crossings divisor in a smooth $W$-scheme $\mathcal{X}$ of arbitrary dimension $n$.

Define the complex of sheaves $Q^{\bullet}$ on $\mathcal{X}$ so that the sequence

$$
0 \rightarrow \Omega_{\mathcal{X}}^{\bullet}(\log \mathcal{D}) \rightarrow \Omega_{\mathcal{X}}^{\bullet}((k+\bullet) \mathcal{D}) \rightarrow Q^{\bullet} \rightarrow 0
$$

is exact.

COROllary 3.3. The homology sheaves $\mathcal{H}^{i}\left(Q^{\bullet}\right)$ of the complex $Q^{\bullet}$ are killed by multiplication by $\operatorname{lcm}\{1,2, \ldots, k+1\}$.

Proof. The long exact sequence for homology sheaves from the short exact sequence (3) has the form

$$
\begin{aligned}
\cdots & \rightarrow \mathcal{H}^{i}\left(\Omega_{\mathcal{X}}^{\bullet}(\log \mathcal{D})\right) \stackrel{\theta_{i}}{\rightarrow} \mathcal{H}^{i}\left(\Omega_{\mathcal{X}}^{\bullet}((k+\bullet) \mathcal{D})\right) \rightarrow \mathcal{H}^{i}\left(Q^{\bullet}\right) \\
& \rightarrow \mathcal{H}^{i+1}\left(\Omega_{\mathcal{X}}^{\bullet}(\log \mathcal{D})\right) \stackrel{\theta_{i+1}}{\rightarrow} \mathcal{H}^{i}\left(\Omega_{\mathcal{X}}^{\bullet}((k+\bullet) \mathcal{D})\right) \rightarrow \cdots
\end{aligned}
$$

Since $\theta_{i+1}$ is an injection by Theorem 3.1, $\mathcal{H}^{i}\left(Q^{\bullet}\right)$ is isomorphic to the cokernel of $\theta_{i}$ and thus by that theorem is killed as claimed.

In the nicest situation one has the following corollary to Theorem 3.1.

COROLLARY 3.4. If the characteristic $p$ of the residue field of $W$ is strictly greater than $k+1$, then we have the following isomorphism of hypercohomology groups:

$$
H_{d R}((\mathcal{X}, \mathcal{D})):=\mathbb{H}\left(\Omega_{\mathcal{X}}^{\bullet}(\log \mathcal{D})\right) \cong \mathbb{H}\left(\Omega_{\mathcal{X}}^{\bullet}((k+\bullet) \mathcal{D})\right) .
$$

Proof. Since in this case $\operatorname{lcm}\{1,2, \ldots, k+1\}$ is invertible in $W$, for each $i$ by Theorem 3.1 we have an isomorphism of homology sheaves $\mathcal{H}^{i}\left(\Omega_{\mathcal{X}}^{\bullet}(\log \mathcal{D})\right) \cong \mathcal{H}^{i}\left(\Omega_{\mathcal{X}}^{\bullet}((k+\right.$ -) $\mathcal{D})$ ). These homology sheaves are the first terms in a spectral sequence $E_{2}^{p, q}:=$ $H^{p}\left(\mathcal{X}, \mathcal{H}^{p}(\star)\right)$ computing the hypercohomology of each complex of sheaves $\star[\mathbf{1 0}$, Remark 2.1.6 (i)]. Hence the hypercohomology groups are isomorphic.

In general, one must be satisfied with the next corollary.

COROllary 3.5. Let $\mathbb{H}^{i}$ denote the ith hypercohomology group. The kernel and cokernel of the map $\mathbb{H}^{i}\left(\Omega_{\mathcal{X}}^{\bullet}(\log \mathcal{D})\right) \rightarrow \mathbb{H}^{i}\left(\Omega_{\mathcal{X}}^{\bullet}((k+\bullet) \mathcal{D})\right)$ are killed by multiplication by $p^{\left\lfloor\log _{p}(k+1)\right\rfloor}$.

Proof. In this case by the proof of Corollary 3.4 for each $i$ the homology sheaves $\mathcal{H}^{i}\left(Q^{\bullet}\right)$ are killed by multiplication by $p^{\left\lfloor\log _{p}(k+1)\right\rfloor}$ and hence so is the hypercohomology $\mathbb{H}\left(Q^{\bullet}\right)$. The result follows from the long exact sequence in hypercohomology for the short exact sequence (3).

3.1.3. The hypercohomology of $\Omega_{\mathcal{X}}^{\bullet}((k+\bullet) \mathcal{D})$.

The hypercohomology of $\Omega_{\mathcal{X}}^{\bullet}((k+\bullet) \mathcal{D})$ is accessible because of the following proposition. 
Proposition 3.6. Assume $k \geqslant 0$ is chosen so that the sheaves $\Omega_{\mathcal{X}}^{i}((k+i) \mathcal{D})$ are acyclic for $i=0,1,2$. Then the hypercohomology of $\Omega_{\mathcal{X}}^{\bullet}((k+\bullet) \mathcal{D})$ is canonically isomorphic to the homology of the complex $\Gamma\left(\mathcal{X}, \Omega_{\mathcal{X}}^{\bullet}((k+\bullet) \mathcal{D})\right)$ of rings of global sections:

$$
0 \rightarrow \Gamma\left(\mathcal{X}, \mathcal{O}_{\mathcal{X}}(k \mathcal{D})\right) \rightarrow \Gamma\left(\mathcal{X}, \Omega_{\mathcal{X}}((k+1) \mathcal{D})\right) \stackrel{d}{\rightarrow} \Gamma\left(\mathcal{X}, \Omega_{\mathcal{X}}^{2}((k+2) \mathcal{D})\right) \rightarrow 0 .
$$

Proof. The hypercohomology of $\Omega_{\mathcal{X}}^{\bullet}((k+\bullet) \mathcal{D})$ may be calculated via a spectral sequence, the first terms of which are the $W$-modules $E_{1}^{p, q}:=H^{q}\left(\mathcal{X}, \Omega_{\mathcal{X}}^{p}((k+p) \mathcal{D})\right)$ [10, Remark 2.1.6 (ii)]. Since the sheaves are acyclic, $E_{1}^{p, q}=0$ for $q>0$, and so this spectral sequence degenerates after the first term. Hence the hypercohomology is just the homology of the complex of rings of global sections.

Corollary 3.7. Assume that $k \geqslant 0$ is chosen to satisfy the conditions of Proposition 3.6. Then the natural map

$$
H_{\text {cris }}^{2}((X, D)) \rightarrow \frac{\Gamma\left(\mathcal{X}, \Omega_{\mathcal{X}}^{2}((k+2) \mathcal{D})\right)}{d\left(\Gamma\left(\mathcal{X}, \Omega_{\mathcal{X}}^{1}((k+1) \mathcal{D})\right)\right.}
$$

has kernel and cokernel killed by multiplication by $p^{\left\lfloor\log _{p}(k+1)\right\rfloor}$. In particular, for $p>k+1$ this map is an isomorphism.

Proof. This follows from Corollary 3.5 and Proposition 3.6 and the natural isomorphism $H_{d R}^{\bullet}((\mathcal{X}, \mathcal{D})) \cong H_{c r i s}^{\bullet}((X, D))$.

Assuming that $\mathcal{D}$ is very ample (which would imply that $\mathcal{U}=\mathcal{X} \backslash \mathcal{D}$ is affine), by Serre's vanishing theorem [12, Theorem III.5.2.(b)] the hypothesis of Proposition 3.6 will be satisfied for $k \gg 0$. We shall calculate an explicit value of $k$ which suffices for the case in which $\mathcal{X}$ is a smooth surface in $\mathbb{P}^{3}$ and $\mathcal{D}$ a smooth hyperplane section.

Note 3.8. Under the assumption that $\mathcal{D}$ is very ample, the twisted logarithmic de Rham complex whose $i$ th term is $\Omega_{\mathcal{X}}^{i}(\log \mathcal{D}) \otimes_{\mathcal{O}_{\mathcal{X}}} \mathcal{O}_{\mathcal{X}}(k \mathcal{D})$, appearing in $[\mathbf{1}$, Theorem 2.2.5], will also be acyclic for $k \gg 0$. However, the complex $\Omega_{\mathcal{X}}^{\bullet}((k+\bullet) \mathcal{D})$ seems easier to compute with.

\section{2. $\quad$ More specific theorems for smooth surfaces in $\mathbb{P}^{3}$}

Let $\mathcal{X}$ be a surface of degree $d$ in $\mathbb{P}:=\mathbb{P}_{W}^{3}$ such that the morphism $\mathcal{X} \rightarrow \operatorname{Spec}(W)$ is smooth, and let $\mathcal{D}$ be a hyperplane section such that the morphism $\mathcal{D} \rightarrow \operatorname{Spec}(W)$ is smooth. Define $\mathcal{U}:=\mathcal{X} \backslash \mathcal{D}$. Let $X, D, U$ (respectively $\mathcal{X}_{K}, \mathcal{D}_{K}, \mathcal{U}_{K}$ ) denote the special (respectively generic) fibres of $\mathcal{X}, \mathcal{D}$ and $\mathcal{U}$.

\subsubsection{Vanishing theorems for smooth surfaces in $\mathbb{P}^{3}$}

In this section we calculate explicit values of $k$ which make the sheaves $\Omega_{\mathcal{X}}^{i}((k+i) \mathcal{D})$ acyclic.

Since the morphism $\mathcal{X} \rightarrow \operatorname{Spec}(W)$ is smooth and hence flat, the ideal sheaf $I$ of $\mathcal{X}$ is such that $I \cong \mathcal{O}_{\mathbb{P}}(-d)$. For $k \in \mathbb{Z}$ let $\mathcal{O}_{\mathcal{X}}(k)$ denote the twisting sheaf, and as usual define $\Omega_{\mathcal{X}}^{i}(k):=\Omega_{\mathcal{X}}^{i} \otimes_{\mathcal{O}_{\mathcal{X}}} \mathcal{O}_{\mathcal{X}}(k)$. For any $k \in \mathbb{Z}$ we have $\Omega_{\mathcal{X}}^{i}(k \mathcal{D}):=$ $\Omega_{\mathcal{X}}^{i} \otimes_{\mathcal{O}_{\mathcal{X}}} \mathcal{O}_{\mathcal{X}}(k \mathcal{D}) \cong \Omega_{\mathcal{X}}^{i}(k)$.

The proof of the following theorem was explained to the author by Bjorn Poonen. 
Theorem 3.9 (Poonen). The sheaves $\mathcal{O}_{\mathcal{X}}(k), \Omega_{\mathcal{X}}(k)$ and $\Omega_{\mathcal{X}}^{2}(k)$ are acyclic for $k>d-4, k>\max \{2 d-4,0\}$, and $k>0$, respectively.

Proof. Let $F$ be the defining polynomial for the surface $\mathcal{X}$, and $\pi: \mathcal{X} \hookrightarrow \mathbb{P}$. The proof is based upon the following three exact sequences of sheaves cf. [12, II.8.17, II.8.13]

$$
\begin{gathered}
0 \rightarrow \mathcal{O}_{\mathbb{P}}(-d) \stackrel{\times F}{\rightarrow} \mathcal{O}_{\mathbb{P}} \rightarrow \pi_{*} \mathcal{O}_{\mathcal{X}} \rightarrow 0 \text { on } \mathbb{P}, \\
0 \rightarrow I /\left.I^{2} \rightarrow \Omega_{\mathbb{P}}\right|_{\mathcal{X}} \rightarrow \Omega_{\mathcal{X}} \rightarrow 0 \text { on } \mathcal{X}, \\
0 \rightarrow \Omega_{\mathbb{P}} \rightarrow \mathcal{O}_{\mathbb{P}}(-1)^{\oplus 4} \rightarrow \mathcal{O}_{\mathbb{P}} \rightarrow 0 \text { on } \mathbb{P},
\end{gathered}
$$

and the calculation of the homology of projective 3 -space $\mathbb{P}$ cf. [12, III.5]

$$
\begin{aligned}
& H^{0}\left(\mathbb{P}, \mathcal{O}_{\mathbb{P}}(e)\right)=0 \text { for } e<0, \\
& H^{1}\left(\mathbb{P}, \mathcal{O}_{\mathbb{P}}(e)\right)=H^{2}\left(\mathbb{P}, \mathcal{O}_{\mathbb{P}}(e)\right)=0 \text { for all } e, \\
& H^{3}\left(\mathbb{P}, \mathcal{O}_{\mathbb{P}}(e)\right)=0 \text { for } e>-4 .
\end{aligned}
$$

Since $\mathcal{O}_{\mathbb{P}}(e)$ is locally free and hence flat we may twist $(5)$ by $\mathcal{O}_{\mathbb{P}}(e)$ and take homology to obtain the exact sequences

$$
H^{i}\left(\mathbb{P}, \mathcal{O}_{\mathbb{P}}(e)\right) \rightarrow H^{i}\left(\mathbb{P}, \pi_{*} \mathcal{O}_{\mathcal{X}}(e)\right) \rightarrow H^{i+1}\left(\mathbb{P}, \mathcal{O}_{\mathbb{P}}(e-d)\right) \text { for } i \geqslant 0 .
$$

Since $\pi$ is affine, we have $H^{i}\left(\mathcal{X}, \mathcal{O}_{\mathcal{X}}(e)\right) \cong H^{i}\left(\mathbb{P}, \pi_{*} \mathcal{O}_{\mathcal{X}}(e)\right)$ and from (8) and the homology of $\mathbb{P}$ we see that the latter vanishes for $i>0$ and $e-d>-4$.

Next, restricting $(7)$ to $\mathcal{X}$, twisting by $\mathcal{O}_{\mathcal{X}}(e)$ and taking homology we get exact sequences

$$
H^{i-1}\left(\mathcal{X}, \mathcal{O}_{\mathcal{X}}(e)\right) \rightarrow H^{i}\left(\mathcal{X}, \Omega_{\mathbb{P}}(e) \mid \mathcal{X}\right) \rightarrow H^{i}\left(\mathcal{X}, \mathcal{O}_{\mathcal{X}}(e-1)\right)^{\oplus 4} \text { for } i \geqslant 1 .
$$

Using our result on the vanishing of $H^{i}\left(\mathcal{X}, \mathcal{O}_{\mathcal{X}}(e)\right)$ we immediately deduce

$$
H^{i}\left(\mathcal{X},\left.\Omega_{\mathbb{P}}(e)\right|_{\mathcal{X}}\right)=0 \text { for } i>1 \text { and } e>d-3 .
$$

For $i=1$, we need also determine when the the map

$$
H^{0}\left(\mathcal{X}, \mathcal{O}_{\mathcal{X}}(e-1)\right)^{\oplus 4} \rightarrow H^{0}\left(\mathcal{X}, \mathcal{O}_{\mathcal{X}}(e)\right)
$$

is a surjection. We need to determine explicitly what the spaces are and map is. First, twisting (5) by $\mathcal{O}_{\mathbb{P}}(e)$ and using the fact $H^{1}\left(\mathbb{P}, \mathcal{O}_{\mathbb{P}}(e)\right)=0$ we get that $H^{0}\left(\mathcal{X}, \mathcal{O}_{\mathcal{X}}(e)\right)$ equals the quotient of the space of homogeneous polynomials of degree $e$ modulo those of the form $F G$ where $G$ is homogeneous of degree $e-d$. Second, observe that the map in (9) comes from that in (7), and the $i$ th component is just multiplication by the $i$ th variable $X_{i}$. So the map is surjective as soon as $e>0$; for one can obtain any monomial of degree $e>0$ by multiplying some monomial of degree $e-1$ by one of the $X_{i}$. Thus the map is surjective when $e>0$, and so $H^{1}\left(\mathcal{X},\left.\Omega_{\mathbb{P}}(e)\right|_{\mathcal{X}}\right)=0$ for $e>\max \{0, d-3\}$.

Now observe since $I \cong \mathcal{O}_{\mathbb{P}}(-d)$ we have

$$
I / I^{2} \cong I \otimes \mathcal{O}_{\mathbb{P}} / I \cong \mathcal{O}_{\mathbb{P}}(-d) \otimes \mathcal{O}_{\mathcal{X}}=\mathcal{O}_{\mathcal{X}}(-d) .
$$

So twisting (6) by $\mathcal{O}_{\mathcal{X}}(e)$ and taking homology we get exact sequences

$$
H^{i}\left(\mathcal{X},\left.\Omega_{\mathbb{P}}(e)\right|_{\mathcal{X}}\right) \rightarrow H^{i}\left(\mathcal{X}, \Omega_{\mathcal{X}}(e)\right) \rightarrow H^{i+1}\left(\mathcal{X}, \mathcal{O}_{\mathcal{X}}(e-d)\right) \text { for } i \geqslant 0 .
$$


Thus $H^{i}\left(\mathcal{X}, \Omega_{\mathcal{X}}(e)\right)=0$ for $i>0$ and $e>\max \{0,2 d-4\}$.

Finally, taking top exterior powers in (6) one deduces $\Omega_{\mathcal{X}}^{2} \cong \mathcal{O}_{\mathcal{X}}(d-4)$, which completes the proof.

\subsubsection{Crystalline lattices for smooth surfaces in $\mathbb{P}^{3}$}

The next two theorems are the main results of Section 3.

THEOREM 3.10. Let $\mathcal{X}$ be a surface of degree $d$ in $\mathbb{P}_{W}^{3}$ such that the morphism $\mathcal{X} \rightarrow \operatorname{Spec}(W)$ is smooth, and $\mathcal{D}$ a hyperplane section such that the morphism $\mathcal{D} \rightarrow \operatorname{Spec}(W)$ is smooth. Let $k+1>\max \{2 d-4,0\}$. Then the natural map

$$
H_{c r i s}^{2}((X, D)) \rightarrow \frac{\Gamma\left(\mathcal{X}, \Omega_{\mathcal{X}}^{2}((k+2) \mathcal{D})\right)}{d\left(\Gamma\left(\mathcal{X}, \Omega_{\mathcal{X}}^{1}((k+1) \mathcal{D})\right)\right)}
$$

has kernel and cokernel killed by multiplication by $p^{\left\lfloor\log _{p}(k+1)\right\rfloor}$.

Proof. Immediate from Corollary 3.7 and Theorem 3.9.

TheOREm 3.11. With notation as in Theorem 3.10, let $H(X, k D)$ denote the image of the righthand side of (10) in $H_{\text {rig }}^{2}(U)$, where $U=X \backslash D$. Let $H(X, \log D)$ denote the image of $H_{\text {cris }}^{2}((X, D))$ in $H_{\text {rig }}^{2}(U)$. Then there is a natural embedding $H(X, \log D) \rightarrow H(X, k D)$ with cokernel killed by multiplication by $p^{\left\lfloor\log _{p}(k+1)\right\rfloor}$.

Proof. The maps $H_{c r i s}^{2}((X, D)) \rightarrow H(X, \log D)$ and

$$
\frac{\Gamma\left(\mathcal{X}, \Omega_{\mathcal{X}}^{2}((k+2) \mathcal{D})\right)}{d\left(\Gamma\left(\mathcal{X}, \Omega_{\mathcal{X}}^{1}((k+1) \mathcal{D})\right)\right)} \rightarrow H(X, k D)
$$

are isomorphisms modulo torsion. The map $H(X, \log D) \rightarrow H(X, k D)$ is defined via these isomorphisms, and its cokernel is killed as claimed by Theorem 3.10. It is am embedding since the kernel of the map (10) is torsion.

\subsection{Frobenius-Hodge structures for smooth surfaces in $\mathbb{P}^{3}$}

In this section we gather some results which will be applied in Sections 4.2 and 5. We begin with an entirely utilitarian definition whose relevance will shortly become apparent. Note that it is exactly adequate for our purposes but inadequate for general use.

Definition 3.12. A torsion-free F-crystal $H$ has a (pure) Frobenius-Hodge structure if there exists $j, m \in \mathbb{Z}$ with $j, m \geqslant 0$ and a filtration by submodules $0=$ $H_{m+1} \subseteq H_{m} \subseteq \cdots \subseteq H_{0}=H$ such that $F\left(H_{i}\right) \subseteq p^{j+i}(H)$ for $0 \leqslant i \leqslant m$. A torsion-free $F$-crystal $H$ has a (mixed) Frobenius-Hodge structure if there exists a short exact sequence of F-crystals $0 \rightarrow H^{\prime} \rightarrow H \rightarrow H^{\prime \prime} \rightarrow 0$ such that $H^{\prime}$ and $H^{\prime \prime}$ have pure Frobenius-Hodge structures.

Let $\mathcal{X}$ be a hypersurface in $\mathbb{P}_{W}^{n}$ such that the morphism $\mathcal{X} \rightarrow \operatorname{Spec}(W)$ is smooth. Denote the special fibre $X$ and generic fibre $\mathcal{X}_{K}$. Then the $W$-modules $H^{j}\left(\mathcal{X}, \Omega_{\mathcal{X}}^{i}\right)$ are torsion free [22, Page 660, Lines 15-16], and thus so are $H_{c r i s}^{m}(X)$ (this is implied by the remarks [22, Page 662, Lines 7-9] and the statement of [22, Theorem 2]). We define the Hodge numbers $h^{i, j}:=\operatorname{rank}\left(H^{j}\left(\mathcal{X}, \Omega_{\mathcal{X}}^{i}\right)\right)=\operatorname{dim}\left(H^{j}\left(\mathcal{X}_{K}, \Omega_{\mathcal{X}_{K}}^{i}\right)\right)$. Note that $h^{i, j}=h^{j, i}$. Let $p$ be the residue characteristic of $W$, and assume that 
$p>n-1=\operatorname{dim}(X)$. The following result is from [22, Section 5]. There exist a filtration (Hodge filtration)

$$
0:=H_{m+1, m} \subseteq H_{m, m} \subseteq H_{m-1, m} \subseteq \cdots \subseteq H_{0, m}=H_{c r i s}^{m}(X)
$$

such that the $p$ th power Frobenius $F$ acts with $F\left(H_{i, m}\right) \subseteq p^{i} H_{c r i s}^{m}(X)$. Moreover, $\operatorname{rank}\left(H_{i, m} / H_{i+1, m}\right)=h^{i, m-i}$ for $0 \leqslant i \leqslant m$. Thus $H_{c r i s}^{m}(X)$ has a pure Frobenius-Hodge structure, see Definition 3.12, with the ranks of the submodules in the filtration determined by the Hodge numbers of $\mathcal{X}_{K}$.

Now let $\mathcal{X}$ be a surface in $\mathbb{P}_{W}^{3}$ such that the morphism $\mathcal{X} \rightarrow \operatorname{Spec}(W)$ is smooth. Let $\mathcal{D} \subset \mathbb{P}_{W}^{2}$ be a hyperplane section such that $\mathcal{D} \rightarrow \operatorname{Spec}(W)$ is smooth. Then the results in the above paragraph apply to give pure Frobenius-Hodge structures on $H_{c r i s}^{2}(\mathcal{X})$ and $H_{c r i s}^{1}(\mathcal{D})$. Since $H_{c r i s}^{1}(X)=H_{c r i s}^{3}(X)$ are torsion-free, and we know $H_{\text {rig }}^{1}(X)=H_{\text {rig }}^{3}(X)=0$ by the Lefschetz hyperplane theorem, one deduces $H_{\text {cris }}^{1}(X)=H_{\text {cris }}^{3}(X)=0$. Moreover, from (1) one deduces, using a similar argument to that in the first paragraph of the proof of Proposition 6.1, that the following sequence is exact.

$$
0 \rightarrow W(-1) \rightarrow H_{\text {cris }}^{2}(X) \rightarrow H_{\text {cris }}^{2}((X, D)) \rightarrow H_{\text {cris }}^{1}(D)(-1) \rightarrow 0 .
$$

Note that $F$ acts by multiplication by $p$ on the rank $1 F$-crystal $W(-1)$, so $H_{\text {cris }}^{2}(X) / W(-1)$ has a pure Frobenius-Hodge structure. The sequence (11) shows that $H_{c r i s}^{2}((X, D))$ is torsion-free, and defines a mixed Frobenius-Hodge structure on $H_{\text {cris }}^{2}((X, D))$, see Definition 3.12 .

\section{Weighted projective surfaces}

In this section we consider the case in which $\mathcal{X}$ is a surface of degree $d$ in the weighted projective space $\mathbb{P}(1, a, b, c)_{W}$. Following a suggestion of A.J. de Jong, we view $\mathcal{X}$ in a standard way as the quotient of a surface $\tilde{\mathcal{X}}$ in $\mathbb{P}_{W}^{3}$ by the action of a finite group $G \cong \mathbb{Z} /(a) \times \mathbb{Z} /(b) \times \mathbb{Z} /(c)$. We shall assume that the residue characteristic $p$ does not divide $a b c$, and that the morphism $\tilde{\mathcal{X}} \rightarrow \operatorname{Spec}(W)$ is smooth. The divisor at infinity $\tilde{\mathcal{D}}$ of $\tilde{\mathcal{X}}$ is $G$-invariant, and we shall assume that the morphism $\tilde{\mathcal{D}} \rightarrow \operatorname{Spec}(W)$ is smooth. Thus the pair $(\tilde{\mathcal{X}}, \tilde{\mathcal{D}})$ satisfies the hypotheses of Theorems 3.10 and 3.11. Define $\tilde{\mathcal{U}}:=\tilde{\mathcal{X}} \backslash \tilde{\mathcal{D}}$. There is a divisor at infinity $\mathcal{D}$ of $\mathcal{X}$ such that $\mathcal{D}=\tilde{\mathcal{D}} / G$ and $\mathcal{U}:=\mathcal{X} \backslash \mathcal{D}$ is affine with $\mathcal{U}=\tilde{\mathcal{U}} / G$. We assume the morphism $\mathcal{U} \rightarrow \operatorname{Spec}(W)$ is smooth. Let $X, D, U, \tilde{X}, \tilde{D}, \tilde{U}$ denote the special fibres, and $\mathcal{X}_{K}, \mathcal{D}_{K}, \mathcal{U}_{K}, \tilde{\mathcal{X}}_{K}, \tilde{\mathcal{D}}_{K}, \tilde{\mathcal{U}}_{K}$ the generic fibres. The rigid cohomology groups $H_{r i g}^{i}(U)$ are defined. Under all these assumptions, we define a lattice $H(X, \log D) \subset H_{\text {rig }}^{2}(U)$ (see Proposition 4.7) and for each $k \geqslant \max \{2 d-4,0\}$ explicitly construct a lattice $H(X, k D) \subset H_{\text {rig }}^{2}(U)$ (Definition 4.9) such that there is a embedding $H(X, \log D) \rightarrow H(X, k D)$ with cokernel killed by multiplication by $p^{\left\lfloor\log _{q}(k+1)\right\rfloor}$ (Theorem 4.10). We explain the importance of the lattices $H(X, \log D)$ and $H(X, k D)$ in Section 5.

\subsection{The lattices $H(X, \log D) \subseteq H(X, k D)$ in $H_{\text {rig }}^{2}(U)$}

We first define our ambient weighted projective space.

Definition 4.1. Let $a, b, c$ be positive integers, and $\mathbb{P}(1, a, b, c)_{W}$ be a weighted projective space over $W$; that is, $\operatorname{Proj}(S)$ where the ring $S:=W[T, X, Y, Z]$ is graded 
by assigning weights $\operatorname{wt}(T):=1, \operatorname{wt}(X):=a, \operatorname{wt}(Y):=b, \operatorname{wt}(Z):=c$. Assume that the residue characteristic $p$ of $W$ does not divide abc.

Note that if desired one can assume that $\operatorname{gcd}(a, b, c)=1$ (although this plays no part in our proofs) since in any case $\mathbb{P}(1, a, b, c)_{W} \cong \mathbb{P}(1, a / e, b / e, c / e)_{W}$ where $e:=\operatorname{gcd}(a, b, c)[\mathbf{8}$, Page 186].

The open subscheme defined by $T \neq 0$ is isomorphic to affine 3 -space $\mathbb{A}_{W}^{3}$ with coordinates given by $x:=X / T^{a}, y:=Y / T^{b}$ and $z:=Z / T^{c}$. Let $R:=$ $W\left[X_{0}, X_{1}, X_{2}, X_{3}\right]$ be the graded polynomial $\operatorname{ring}$ with $\operatorname{wt}\left(X_{i}\right):=1$ for $0 \leqslant i \leqslant 3$ and define projective 3 -space $\mathbb{P}_{W}^{3}:=\operatorname{Proj}(R)$. Consider the injective map of graded rings

$$
\pi: S \rightarrow R, T \mapsto X_{0}, X \mapsto X_{1}^{a}, Y \mapsto X_{2}^{b}, Z \mapsto X_{3}^{c}
$$

Assume now that $W$ is large enough to contain primitive $a$ th, $b$ th and $c$ th roots of unity $\zeta_{a}, \zeta_{b}$ and $\zeta_{c}$, respectively. See Note 4.11 for a discussion of the general case. Let the group $G:=\mathbb{Z} /(a) \times \mathbb{Z} /(b) \times \mathbb{Z} /(c)$ act on $R$ by

$$
G \times R \rightarrow R,(i, j, k) \times f\left(X_{0}, X_{1}, X_{2}, X_{3}\right) \mapsto f\left(X_{0}, \zeta_{a}^{i} X_{1}, \zeta_{b}^{j} X_{2}, \zeta_{c}^{k} X_{3}\right) .
$$

Lemma 4.2. The invariant subring $R^{G}$ of $R$ under the action of $G$ is exactly $\pi(S)=$ $R\left[X_{0}, X_{1}^{a}, X_{2}^{b}, X_{3}^{c}\right]$.

Proof. Certainly $\pi(S) \subseteq R^{G}$. Suppose now that $f=\sum_{i} a_{i} X^{i} \in R^{G}$ where $i:=$ $\left(i_{0}, i_{1}, i_{2}, i_{3}\right) \in \mathbb{Z}_{\geqslant 0}^{4}$ and $X^{i}:=X_{0}^{i_{0}} \cdots X_{3}^{i_{3}}$. Since $G$ acts on each term $a_{i} X^{i}$ in $f$ by scalar multiplication, each term in $f$ must be fixed by $G$. So it is enough to consider the case of a single monomial $f=X_{0}^{i_{0}} \cdots X_{3}^{i_{3}}$. By considering the action of $(1,0,0),(0,1,0),(0,0,1) \in G$ in turn, one deduces that $a\left|i_{1}, b\right| i_{2}$ and $c \mid i_{3}$, so $f \in \pi(S)$, as required.

COROLlary 4.3. The weighted projective space $\mathbb{P}(1, a, b, c)_{W}$ is isomorphic to the quotient scheme $\mathbb{P}_{W}^{3} / G$.

Proof. By Lemma 4.2, $S \stackrel{\pi}{\cong} R^{G}$, and so $\operatorname{Proj}(S) \cong \operatorname{Proj}\left(R^{G}\right)=: \mathbb{P}_{W}^{3} / G$.

Let $\mathcal{X}$ be a hypersurface in $\mathbb{P}(1, a, b, c)_{W}$ defined by a weighted homogeneous polynomial $P(T, X, Y, Z)$ of degree $d$. Assume that the hypersurface $\tilde{\mathcal{X}}$ in $\mathbb{P}_{W}^{3}$ defined by the polynomial $\pi(P)=P\left(X_{0}, X_{1}^{a}, X_{2}^{b}, X_{3}^{c}\right)$ is such that the morphism $\tilde{\mathcal{X}} \rightarrow \operatorname{Spec}(W)$ is smooth. Define $\mathcal{D}$ and $\tilde{\mathcal{D}}$ to be the divisors on $\mathcal{X}$ and $\tilde{\mathcal{X}}$, respectively, defined by the ideals $(T)$ and $\left(X_{0}\right)$, respectively. Assume that the morphism $\tilde{\mathcal{D}} \rightarrow \operatorname{Spec}(W)$ is smooth. Let $\mathcal{U}:=\mathcal{X} \backslash \mathcal{D}$ and $\tilde{\mathcal{U}}:=\tilde{\mathcal{X}} \backslash \tilde{\mathcal{D}}$. Assume that the morphism $\mathcal{U} \rightarrow \operatorname{Spec}(W)$ is smooth. Let $X, D, U, \tilde{X}, \tilde{D}, \tilde{U}$ denote the special fibres, and $\mathcal{X}_{K}, \mathcal{D}_{K}, \mathcal{U}_{K}, \tilde{\mathcal{X}}_{K}, \tilde{\mathcal{D}}_{K}, \tilde{\mathcal{U}}_{K}$ the generic fibres.

Lemma 4.4. The group $G$ acts on $\tilde{\mathcal{X}}$ with $\mathcal{X} \cong \tilde{\mathcal{X}} / G$, on $\tilde{\mathcal{U}}$ with $\mathcal{U} \cong \tilde{\mathcal{U}} / G$, and on $\tilde{\mathcal{D}}$ with $\mathcal{D} \cong \tilde{\mathcal{D}} / G$.

Proof. The group $G$ acts on $\tilde{\mathcal{X}}$ since the ideal $(\pi(P))$ is $G$-invariant. Now $\tilde{\mathcal{X}} / G:=$ $\operatorname{Proj}\left((R /(\pi(P)))^{G}\right)$ and we note $(R /(\pi(P)))^{G} \cong R^{G} /(\pi(P))$. The isomorphism $S \stackrel{\pi}{\rightarrow} R^{G}$ reduces modulo the homogeneous ideal $(P)$ to an isomorphism $S /(P) \stackrel{\pi}{\rightarrow}$ $R^{G} /(\pi(P))=(R /(\pi(P)))^{G}$. So $\mathcal{X}=\operatorname{Proj}(S /(P))=\tilde{\mathcal{X}} / G$. 
Since $G$ acts trivially on $T$, it acts on $\tilde{\mathcal{U}}$ with $(i, j, k) \times g(x, y, z) \mapsto g\left(\zeta_{a}^{i} x, \zeta_{b}^{j} y, \zeta_{c}^{k} z\right)$, for $g \in W[x, y, z]=W\left[X / T^{a}, Y / T^{b}, Z / T^{c}\right]$. The proof that $\mathcal{U} \cong \tilde{\mathcal{U}} / G$ is similar to that in the preceding paragraph. The group $G$ acts on $\tilde{\mathcal{D}}$ since it fixes the homogeneous ideal $(T)$, and one sees as before that $\mathcal{D} \cong \tilde{\mathcal{D}} / G$.

LEMma 4.5. For each $i \geqslant 0$, the group $G$ acts on the rigid cohomology space $H_{\text {rig }}^{i}(\tilde{U})$ and $H_{\text {rig }}^{i}(U) \cong H_{\text {rig }}^{i}(\tilde{U})^{G}$.

Proof. Let $A$ and $B$ denote the coordinate rings of $\mathcal{U}_{K}$ and $\tilde{\mathcal{U}}_{K}$, respectively; so $A \cong B^{G}$. Taking weak completions we have $A^{\dagger} \cong\left(B^{G}\right)^{\dagger} \cong\left(B^{\dagger}\right)^{G}$, the latter isomorphism follows since taking invariants of the action of a finite group commutes with taking weak completions. (One can see this explicitly here by first observing $\left(K[x, y, z]^{G}\right)^{\dagger} \cong\left(K[x, y, z]^{\dagger}\right)^{G}$, and then fixing a monomial ordering and identifying the quotient $B$ of $K[x, y, z]$ as a $K$-vector space with the space spanned in $K[x, y, z]$ by a basis of monomials [5, Proposition 5.3.4].) Let $d: B^{\dagger} \rightarrow \Omega_{B^{\dagger}}$ be the universal derivation which is continuous w.r.t. the $p$-adic metric on $B^{\dagger}$. One checks that $\left(\Omega_{B^{\dagger}}^{\bullet}\right)^{G}$ is the direct factor in $\Omega_{B^{\dagger}}^{\bullet}$ corresponding to the trivial character in a character-based decomposition, that $\left(\Omega_{B^{\dagger}}^{\bullet}\right)^{G} \cong \Omega_{\left(B^{\dagger}\right)^{G}}^{\bullet}$ as complexes of $\left(B^{\dagger}\right)^{G}$ modules, and since $\left(B^{\dagger}\right)^{G} \cong A^{\dagger}$ we have $\Omega_{\left(B^{\dagger}\right)^{G}}^{\bullet} \cong \Omega_{A^{\dagger}}^{\bullet}$. The rigid cohomology of $\tilde{U}$ is by definition the homology $H\left(\Omega_{B^{\dagger}}^{\bullet}\right.$. Since $\left(\Omega_{B^{\dagger}}^{\bullet}\right)^{G}$ is the trivial-character direct factor in $\Omega_{B^{\dagger}}^{\bullet}$ one has $H\left(\Omega_{B^{\dagger}}^{\bullet}\right)^{G} \cong H\left(\left(\Omega_{B^{\dagger}}^{\bullet}\right)^{G}\right)$, and we know $H\left(\left(\Omega_{B^{\dagger}}^{\bullet}\right)^{G}\right) \cong H\left(\Omega_{A^{\dagger}}\right)$. But the latter is by definition the rigid cohomology of $U$.

For each $0 \leqslant i \leqslant 4$, the log-crystalline cohomology group $H_{\text {cris }}^{i}((\tilde{X}, \tilde{D}))$ is defined, and there is a natural map

$$
H_{\text {cris }}^{i}((\tilde{X}, \tilde{D})) \rightarrow H_{r i g}^{i}(\tilde{U})
$$

which is an injection modulo torsion with image a full-rank $W$-lattice. These morphisms commute with the action of $G$, so we may take $G$-invariants to get natural maps

$$
H_{c r i s}^{i}((\tilde{X}, \tilde{D}))^{G} \rightarrow H_{r i g}^{i}(\tilde{U})^{G}
$$

and by Lemma 4.5 the codomain is isomorphic to $H_{r i g}^{i}(U)$.

Definition 4.6. For each $\underset{\tilde{D}}{i} \geqslant 0$, define the $W$-module $H_{\text {cris }}^{i}((X, D))$ to be the $G$-invariant part $H_{\text {cris }}^{i}((\tilde{X}, \tilde{D}))^{G}$. We shall call these groups the log-crystalline cohomology of the pair $(X, D)$.

Proposition 4.7. There is a natural map which is an injection modulo torsion $H_{\text {cris }}^{i}((X, D)) \rightarrow H_{\text {rig }}^{i}(U)$, with image a full-rank $F$-invariant $W$-lattice. For $i=2$, let us denote this lattice $H(X, \log D)$.

Proof. We constructed the natural map immediately before Definition 4.6, and it is an injection modulo torsion. It is $F$-invariant since $F$ permutes the eigenspaces of the $G$-action and preserves the trivial eigenspace. Since the order of $G$ is coprime to $p$, there is a $G$-invariant decomposition

$$
H_{\text {cris }}^{i}((\tilde{X}, \tilde{D}))=H_{\text {cris }}^{i}((\tilde{X}, \tilde{D}))^{G} \oplus C_{\text {cris }} \rightarrow H_{\text {rig }}^{i}(\tilde{U})^{G} \oplus C_{\text {rig }}=H_{\text {rig }}^{i}(\tilde{U})
$$

and a natural map which is an injection modulo torsion $C_{\text {cris }} \rightarrow C_{\text {rig }}$. If the map in the proposition did not have a full-rank image, then the rank of the image of $C_{c r i s}$ in $C_{r i g}$ would have to exceed the dimension of $C_{\text {rig }}$, which is impossible. 
Note 4.8. The $\mathbb{F}_{q}$-variety $X$ in general will only be quasi-smooth, that is have quotient singularities, which in our case lie along the divisor $D[\mathbf{9}$, Appendix B]. So the log-crystalline cohomology $H_{c r i s}^{i}((X, D))$ is not a priori defined. The divisor $D$ itself is a quasi-smooth curve in weighted projective space $\mathbb{P}(a, b, c)_{\mathbb{F}_{q}}$. Quasismooth varieties are normal, and hence the curve $D$ itself is smooth. The author believes that $H_{c r i s}^{2}((X, D))$ defined above is isomorphic to $H_{c r i s}^{2}\left(\left(X_{1}, D_{1}\right)\right)$ where $X_{1}$ is some smooth compactification of $U$ with $D_{1}:=X_{1} \backslash U_{1}$ a smooth normal crossing divisor; however, he offers no proof of this.

Using Theorem 3.10, the group $H_{c r i s}^{2}((\tilde{X}, \tilde{D}))$ can be explicitly related to the cohomology classes in $H_{\text {rig }}^{2}(\tilde{U})$ spanned by a specific set of elements in $\Gamma\left(\tilde{\mathcal{U}}, \Omega_{\tilde{\mathcal{U}}}^{2}\right)$. Since $H_{\text {cris }}^{2}((X, D)):=H_{\text {cris }}^{2}((\tilde{X}, \tilde{D}))^{G}$ we can construct a group "close to" $H_{\text {cris }}^{2}((X, D))$ by taking the $G$-invariants of this set. Explicitly, for $k+1>\max \{2 d-4,0\}$ we see that the map

$$
H_{\text {cris }}^{2}((X, D)) \rightarrow \frac{\Gamma\left(\tilde{\mathcal{X}}, \Omega_{\tilde{\mathcal{X}}}^{2}((k+2) \tilde{\mathcal{D}})\right)^{G}}{d\left(\Gamma\left(\tilde{\mathcal{X}}, \Omega_{\tilde{\mathcal{X}}}^{1}((k+1) \tilde{\mathcal{D}})\right)\right)^{G}}=\frac{\Gamma\left(\mathcal{X}, \Omega_{\mathcal{X}}^{2}((k+2) \mathcal{D})\right)}{d\left(\Gamma\left(\mathcal{X}, \Omega_{\mathcal{X}}^{1}((k+1) \mathcal{D})\right)\right)}
$$

has kernel and cokernel killed by multiplication by $p^{\left\lfloor\log _{p}(k+1)\right\rfloor}$. The last equality of $W$-modules in (12) follows from the isomorphism $\Gamma\left(\mathcal{U}, \Omega_{\mathcal{U}}^{i}\right) \cong \Gamma\left(\mathcal{U},\left(\rho_{*} \Omega_{\tilde{\mathcal{U}}}^{i}\right)^{G}\right)$ where $\rho: \tilde{\mathcal{U}} \rightarrow \mathcal{U}$ is the quotient map, cf. [25, Page 215, Lines 8-12], and the fact that pole orders are just calculated by taking suitable weighted degrees.

Definition 4.9. For each $k+1 \geqslant \max \{2 d-4,0\}$, the lattice $H(X, k D)$ is defined to be the image of the righthand side of (12) in $H_{\text {rig }}^{2}(U)$.

THEOREM 4.10. There is a natural embedding $H(X, \log D) \rightarrow H(X, k D)$ with cokernel killed by multiplication by $p^{\left.\log _{p}(k+1)\right\rfloor}$.

Proof. The two groups are subgroups of $H_{\text {rig }}^{2}(U)$, and the map which defines $H(X, k D)$ factors through that which defines $H(X, \log D)$, so there is an obvious inclusion. The statement on the cokernel comes from the remark immediately following (12).

The $K$-vector space $H(X, k D) \otimes_{W} K$ is $F$-invariant and so has the structure of an $F$-isocrystal; however, $H(X, k D)$ itself is not in general $F$-invariant.

Note 4.11. In the general case one extends the base field to contain appropriate primitive roots and takes the Galois invariant part to define the lattice $H(X, \log D)$ in $H_{\text {rig }}^{2}(U)$. The lattice $H(X, k D)$ can be defined explicitly over $W$ and Theorem 4.10 remains true.

Note 4.12. Direct calculations for surfaces in weighted projective space (ignoring the technical problems raised by singularities) and experiments suggest that in fact $k+1>\max \{2 d-(1+a+b+c), 0\}$ is sufficient for the map in (12) to have kernel and cokernel killed as claimed. If this calculation could be made rigorous one would avoid consideration of the surface $\tilde{\mathcal{X}}$ altogether, and in particular remove the additional, and in practice irrelevant, assumption that the morphism $\tilde{\mathcal{X}} \rightarrow \operatorname{Spec}(W)$ is smooth. 


\subsection{Frobenius-Hodge structures for weight projective surfaces}

We continue with the notation and assumptions in Section 4.1 - these are stated concisely in the paragraph immediately preceding it. We shall also use the notation in the final paragraph of Section 3.3, only we now denote our projective surface and curve as $\tilde{\mathcal{X}}$ and $\tilde{\mathcal{D}}$, respectively, rather than $\mathcal{X}$ and $\mathcal{D}$. Taking $G$-invariants in the exact sequence $(11)$ for the pair $(\tilde{X}, \tilde{D})$ and noting the first non-zero term is $H_{c r i s}^{0}(\tilde{D})$ we get the sequence

$$
0 \rightarrow W(-1) \rightarrow H_{\text {cris }}^{2}(\tilde{X})^{G} \rightarrow H_{\text {cris }}^{2}((X, D)) \rightarrow H_{\text {cris }}^{1}(\tilde{D})(-1)^{G} \rightarrow 0 .
$$

Since Frobenius $F$ preserves the trivial eigenspace of the $G$-action, the above exact sequence defines a mixed Frobenius-Hodge structure (Definition 3.12) on $H_{c r i s}^{2}((X, D))$. We now examine this in more detail.

Let us first recall how one defines Hodge numbers of quasi-smooth varieties, cf. [4, Appendix A.3].

Definition 4.13. Let $j: \mathcal{X}_{K}^{s} \rightarrow \mathcal{X}_{K}$ be the embedding of the smooth locus and $i \geqslant 0$, and define the sheaf $\hat{\Omega}_{\mathcal{X}_{K}}^{i}:=j_{*} \Omega_{\mathcal{X}_{K}^{s}}^{i}$ on $\mathcal{X}_{K}$. The Hodge numbers $h^{i, j}$ are the dimensions of the $K$-vector spaces $H^{j}\left(\mathcal{X}_{K}, \hat{\Omega}_{\mathcal{X}_{K}}^{i}\right)$.

Note that $\mathcal{D}_{K}$ is smooth so its Hodge numbers are defined in the usual manner, as the dimensions of the spaces $H^{j}\left(\mathcal{D}_{K}, \Omega_{\mathcal{D}_{K}}^{i}\right)$. By the result on [25, Page 215, Lines 8-12], for each $i \geqslant 0$ we have an isomorphism of sheaves $\left(\rho_{*} \Omega_{\tilde{\mathcal{D}}_{K}}^{i}\right)^{G} \cong \Omega_{\mathcal{D}_{K}}^{i}$ and $\left(\rho_{*} \Omega_{\tilde{\mathcal{X}}_{K}}^{i}\right)^{G} \cong \hat{\Omega}_{\mathcal{X}_{K}}^{i}$ where $\rho: \tilde{\mathcal{X}}_{K} \rightarrow \mathcal{X}_{K}$ is the quotient map and also its restriction $\rho: \tilde{\mathcal{D}}_{K} \rightarrow \mathcal{D}_{K}$. It follows by decomposing sheaves via characters of $G$ and using the finiteness of $\rho$ that $H^{j}\left(\tilde{\mathcal{X}}_{K}, \Omega_{\tilde{\mathcal{X}}_{K}}^{i}\right)^{G} \cong H^{j}\left(\mathcal{X}_{K}, \hat{\Omega}_{\mathcal{X}_{K}}^{i}\right)$ and $H^{j}\left(\tilde{\mathcal{D}}_{K}, \Omega_{\tilde{\mathcal{D}}_{K}}^{i}\right)^{G} \cong$ $H^{j}\left(\mathcal{D}_{K}, \Omega_{\mathcal{D}_{K}}^{i}\right)$. Thus the dimensions of the quotients in the filtrations on $H_{c r i s}^{2}(\tilde{X})^{G}$ and $H_{\text {cris }}^{1}(\tilde{D})^{G}$ are just the Hodge numbers of the quasi-smooth surface $\mathcal{X}_{K}$ and smooth curve $\mathcal{D}_{K}$.

Since $H_{\text {cris }}^{2}((X, D))$ is a submodule of $H_{c r i s}^{2}((\tilde{X}, \tilde{D}))$ and the latter is torsion free, so is the former. Thus the lattice $H(X, \log D):=\operatorname{Im}\left(H_{\text {cris }}^{2}((X, D)) \rightarrow H_{\text {rig }}^{2}(U)\right)$ is isomorphic to $H_{c r i s}^{2}((X, D))$. Defining $H(X)_{\text {prim }}:=H_{c r i s}^{2}(\tilde{X})^{G} / W(-1)$ we see that there is a filtration

$$
0 \subseteq H_{2} \subseteq H_{1} \subseteq H_{0}=H(X)_{\text {prim }}
$$

with $F\left(H_{i}\right) \subseteq p^{i} H(X)_{\text {prim }}$. Recall $h^{i, j}:=\operatorname{dim}\left(H^{j}\left(\mathcal{X}_{K}, \hat{\Omega}_{\mathcal{X}_{K}}^{i}\right)\right)$ are the Hodge numbers of the quasi-smooth variety $\mathcal{X}_{K}$. We have $h^{2,0}=\operatorname{rank} H_{2}$ and $h^{1,1}-1=$ $\operatorname{rank}\left(H_{1} / H_{2}\right)$ (we have removed the hyperplane class), and $h^{0,2}=\operatorname{rank}\left(H_{0} / H_{1}\right)$. Defining $h_{2}:=h^{2,0}+h^{1,1}+h^{0,2}$, the middle Betti number of $\mathcal{X}_{K}$, we see that $H(X)_{\text {prim }}$ has rank $h_{2}-1$.

Likewise, the lattice

$$
H(D):=\operatorname{Im}\left(H_{\text {cris }}^{1}(\tilde{D})^{G} \rightarrow H_{\text {rig }}^{1}(\tilde{D})^{G}\right)
$$

is isomorphic to $H_{c r i s}^{1}(\tilde{D})^{G}$ and has a filtration

$$
0 \subseteq H_{1}^{\prime} \subseteq H_{0}^{\prime}=H(D)
$$

with $F\left(H_{i}^{\prime}\right) \subseteq p^{i}(H(D))$ and $\operatorname{rank} H_{1}^{\prime}=g=\operatorname{rank}\left(H_{0}^{\prime} / H_{1}^{\prime}\right)$ where the genus $g:=$ $\operatorname{dim}\left(H^{0}\left(\mathcal{D}_{K}, \Omega_{\mathcal{D}_{K}}^{1}\right)\right)$. 
So the mixed Frobenius-Hodge structure on $H(X, \log D) \cong H_{\text {cris }}^{2}((X, D))$ comes from the exact sequence

$$
0 \rightarrow H(X)_{\text {prim }} \rightarrow H(X, \log D) \rightarrow H(D)(-1) \rightarrow 0
$$

and the dimensions of the quotients in the filtrations on $H(X)_{\text {prim }}$ and $H(D)(-1)$ are the Hodge numbers of the quasi-smooth surface $\mathcal{X}_{K}$ and smooth curve $\mathcal{D}_{K}$.

\subsection{Surfaces of the form $z^{2}=Q(x, y)$}

We now describe an explicit method for constructing the lattice $H(X, k D)$ (Definition 4.9) in the case in which $\mathcal{U}$ is a surface defined by a polynomial $z^{2}=Q(x, y)$.

\subsubsection{Differential forms on the surface}

Assume that the characteristic $p$ of the residue field of $W$ is odd. Let $a, b, c$ be positive integers with $\operatorname{gcd}(a, b)=1$ and $a b c$ not divisible by $p$. Let $Q(x, y)=$ $\sum_{i, j} q_{i, j} x^{i} y^{j}$ be a polynomial in $W[x, y]$ such that $a i+b j \leqslant 2 c$ for all pairs $(i, j)$, with this bound met for some choice of pair $(i, j)$; that is, $Q(x, y)$ has weighted degree $2 c$ when one assigns weights $\operatorname{wt}(x):=a$ and $\operatorname{wt}(y):=b$ in the polynomial ring $W[x, y]$. Let $\mathcal{X}$ be the hypersurface of degree $d:=2 c$ in $\mathbb{P}(1, a, b, c)_{W}$ defined by the polynomial

$$
P:=T^{d}\left(\left(Z / T^{c}\right)^{2}-Q\left(X / T^{a}, Y / T^{b}\right)\right) .
$$

Let $\mathcal{D}$ denote the scheme defined by the ideal $(P, T)$ and $\mathcal{U}:=\mathcal{X} \backslash \mathcal{D}$. The affine $W$ scheme $\mathcal{U}$ is defined by the polynomial $z^{2}=Q(x, y)$, where $x=X / T^{a}, y=Y / T^{b}$ and $z:=Z / T^{c}$. We assume that the morphism $\mathcal{U} \rightarrow \operatorname{Spec}(W)$ is smooth. We further assume that the schemes $\tilde{\mathcal{X}}$ and $\tilde{\mathcal{D}}$, respectively, in $\mathbb{P}_{W}^{3}$ defined by the polynomial $\pi(P):=P\left(X_{0}, X_{1}^{a}, X_{2}^{b}, X_{3}^{c}\right)$ and ideal $\left(\pi(P), X_{0}\right)$, respectively, are such that the morphisms $\tilde{\mathcal{X}} \rightarrow \operatorname{Spec}(W)$ and $\tilde{\mathcal{D}} \rightarrow \operatorname{Spec}(W)$ are smooth, although this will play no role in the construction of the lattice $H(X, k D)$.

LEMMA 4.14. The functions $x, y$, and $z$ have poles of orders $a, b$ and $c$, respectively, along the divisor $\mathcal{D}$. The 1 -forms $d x, d y$ and $d z$ have poles of orders $a+1, b+1$ and $c+1$ along $\mathcal{D}$, and the 2 -form $d x \wedge d y$ a pole of order $a+b+2$ along $\mathcal{D}$.

Proof. Follows since the divisor $\mathcal{D}$ is defined by the homogeneous ideal $(T)$, differentiation increases pole orders by one, and $d x \wedge d y=d(y d x)$.

By definition, the module $\Gamma\left(\mathcal{U}, \Omega_{\mathcal{U}}^{1}\right)$ is generated over $W[x, y, z] /\left(z^{2}-Q(x, y)\right)$ by the 1 -forms $d x, d y$ and $d z$ subject to the one relation

$$
2 z d z=Q_{x} d x+Q_{y} d y .
$$

It follows immediately that the module $\Gamma\left(\mathcal{U}, \Omega_{\mathcal{U}}^{2}\right)$ is generated over $W[x, y, z] /\left(z^{2}-\right.$ $Q(x, y))$ by the 2 -forms $d x \wedge d y, d y \wedge d z$ and $d z \wedge d x$ subject to the relations

$$
d y \wedge d z=-\frac{Q_{x}}{2 z} d x \wedge d y, d z \wedge d x=-\frac{Q_{y}}{2 z} d x \wedge d y
$$

Since $\mathcal{U}$ is smooth we have $\left(Q, Q_{x}, Q_{y}\right)=(1)$. A simple computation now reveals that

$$
\Gamma\left(\mathcal{U}, \Omega_{\mathcal{U}}^{2}\right)=\left\{\frac{A+B z}{z} d x \wedge d y \mid A, B \in W[x, y]\right\} .
$$


By Lemma 4.14, the pole order of such a 2-form along $\mathcal{D}$ is bounded above by

$$
\max \{\operatorname{wt}(A)-c, \operatorname{wt}(B)\}+a+b+2
$$

where wt here means weighted degree.

Lemma 4.15. Let $B \in W[x, y]$ have ordinary degree $\operatorname{deg}(B)$. Then for any $k+2 \geqslant$ $\mathrm{wt}(B)+a+b+2$, the class of the 2 -form $B d x \wedge d y$ in the quotient on the righthand side of (12) is killed by multiplication by $p^{\left.\log _{p}(\operatorname{deg}(B))\right\rfloor}$; thus it is the zero class in $H_{\text {rig }}^{2}(U)$.

Proof. Integrating w.r.t. $x$, say, one constructs a polynomial $C \in W[x, y]$ with $d C / d x=p^{\left\lfloor\log _{p}(\operatorname{deg}(B))\right\rfloor} B$. Then $d(C \wedge d y)=p^{\left\lfloor\log _{p}(\operatorname{deg}(B))\right\rfloor} B d x \wedge d y$, as required.

Since we wish to construct the image in $H_{\text {rig }}^{2}(U)$ of the righthand side of $(12)$, by Lemma 4.15 we can restrict attention to 2 -forms $(A / z) d x \wedge d y$.

By an explicit computation one finds that the exact forms $(A / z) d x \wedge d y$ are precisely those for which

$$
2 A=\alpha Q_{x}+\beta Q_{y}+2\left(\alpha_{x}+\beta_{y}\right) Q, \alpha, \beta \in W[x, y] .
$$

Precisely, such a form is $d$ of

$$
C z d x+D z d y+E d z, \alpha:=D-E_{y}, \beta:=-C+E_{x}, C, D, E \in W[x, y] .
$$

Note that the pole order of such a 1 -form is bounded by $\max \{\operatorname{wt}(\alpha)+c+b+$ $1, \operatorname{wt}(\beta)+c+a+1\}$. So to ensure this has pole order at most $k+1$ we need to have $\operatorname{wt}(\alpha) \leqslant(k+1)-(c+b+1)=k-(b+c)$ and $\operatorname{wt}(\beta) \leqslant k-(a+c)$. Moreover, the 2 -forms $(A / z) d x \wedge d y$ have pole order bounded by wt $(A)-c+a+b+2$, so to ensure the pole order is at most $k+2$ it is enough to have $\operatorname{wt}(A) \leqslant(k+2)+c-(a+b+2)=$ $k+c-(a+b)$.

\subsubsection{An algorithm for constructing the lattice $H(X, k D)$}

For any $k \geqslant 0$, one constructs a set which generates the quotient in (12) as follows. For any integer $m \geqslant 0$, let Mon $\leqslant m_{\leqslant}$denote the union of the set of monomials $x^{i} y^{j}$ of weighted degree at most $m$ with $\{0\}$. Let $Z_{m}$ denote the free $W$-submodule of $W[x, y]$ spanned by the monomials in Mon $\leqslant m+c-(a+b+2)$. Let $B_{m} \subseteq Z_{m}$ be the $W$-module generated by polynomials of the form $\alpha Q_{x}+\beta Q_{y}+2\left(\alpha_{x}+\beta_{y}\right) Q$ where $\alpha \in \operatorname{Mon}_{\leqslant(m-1)-(b+c+1)}$ and $\beta \in \operatorname{Mon}_{\leqslant(m-1)-(a+c+1)}$. Define $H_{m}:=Z_{m} / B_{m}$. Then $H_{k+2}$ is isomorphic to the quotient in (12) via the map

$$
\theta: Z_{k+2} \rightarrow \Gamma\left(\mathcal{X}, \Omega_{\mathcal{X}}^{2}((k+2) \mathcal{D})\right), x^{i} y^{j} \mapsto x^{i} y^{j} d x \wedge d y / 2 z .
$$

One can find a set $S(k) \subseteq$ Mon $_{\leqslant k+c-(a+b)}$ of monomials whose classes in $H_{k+2}$ are a $W$-generating set for this quotient using the following method.

Let $Z_{m, p}:=Z_{m} \otimes_{W} \mathbb{F}_{q}$ and $B_{m, p}:=B_{m} \otimes_{W} \mathbb{F}_{q}$ be $\mathbb{F}_{q}$-vector spaces. Using linear algebra over $\mathbb{F}_{q}$, compute a generating set $S(k)$ of monomials for the quotient $Z_{k+2, p} / B_{k+2, p}$. Let $\langle S(k)\rangle_{W}$ denote the $W$-span of this generating set. Let $w \in$ $Z_{k+2}$. Then $w=u_{1}+v_{1} \bmod p$ for some $u_{1} \in\langle S(k)\rangle_{W}$ and $v_{1} \in B_{k+2}$. Write $w=u_{1}+v_{1}+p w_{2}$ where $w_{2} \in Z_{k+2}$. Repeating the process we find $u_{2} \in\langle S(k)\rangle_{W}$ and $v_{2} \in B_{k+2}$ such that $w=\left(u_{1}+p u_{2}\right)+\left(v_{1}+p v_{2}\right)+p^{2} w_{2}$ for some $w_{2} \in Z_{k+2}$. By induction, and taking a $p$-adic limit, we find $u \in\langle S(k)\rangle_{W}$ and $v \in B_{k+2}$ such that $w=u+v$, as required. 
If $p>k+1$ and $k \geqslant \max \{2 d-4,0\}$, and the classes of the forms

$$
\theta(s)=(s / 2 z) d x \wedge d y \text { for } s \in S(k)
$$

are independent in $H_{\text {rig }}^{2}(U)$, then the forms (16) are a basis for the lattice

$$
H(X, \log D)=\operatorname{Im}\left(H_{\text {cris }}^{2}((X, D)) \rightarrow H_{\text {rig }}^{2}(U)\right) .
$$

Note that they will be independent in $H_{r i g}^{2}(U)$ if and only if $|S(k)|=\operatorname{dim} H_{r i g}^{2}(U)$; the latter is just ( $\left.\operatorname{dim} H_{\text {rig }}^{2}(X)-1\right)+\operatorname{dim} H_{\text {rig }}^{1}(D)$ (cf. first paragraph of the proof of Proposition 6.1), and so is easily calculated.

If $k \geqslant \max \{2 d-4,0\}$ and the classes of (16) are independent in $H_{\text {rig }}^{2}(U)$, then the classes of (16) span a lattice $H(X, k D) \subset H_{r i g}^{2}(U)$ such that there is a natural embedding $\rho: H(X, \log D) \rightarrow H(X, k D)$ with cokernel killed by $p^{\left\lfloor\log _{p}(k+1)\right\rfloor}$. If the classes are not independent in $H_{\text {rig }}^{2}(U)$ then one must do some further work to extract a basis for the free part of $\langle S(k)\rangle_{W}$. We have not come across this situation in practice and so do not address it here.

\section{Frobenius-Hodge structures and precision estimates}

In this section we explain how the use of a basis for the full-rank $W$-lattice $H(X, k D)$ in $H_{\text {rig }}^{2}(U)$ when calculating the Frobenius action allows one to compute with lower $p$-adic precisions. Notation and assumptions are as summarised in the first paragraph of Section 4.

To state the next theorem in a simple manner, we shall say informally that a rational approximation $\tilde{a} \in \mathbb{Q}$ to a $p$-adic number $a \in \mathbb{Q}_{p}$ is correct modulo $p^{N}$ if $\operatorname{ord}_{p}(\tilde{a}-a) \geqslant N$, with this notion extended in the obvious way to matrices and polynomials.

TheOREM 5.1. Assume that the residue field of $W$ is the prime field $\mathbb{F}_{p}$ where $p>2$, and denote by $h^{2,0}:=\operatorname{dim} H^{0}\left(\mathcal{X}_{K}, \hat{\Omega}_{\mathcal{X}_{K}}^{2}\right)$ the geometric genus of $\mathcal{X}_{K}$ (Definition 4.13). Let $\tilde{A}$ be an approximation to the matrix for the Frobenius map $F$ on $H(X, k D) \otimes_{W} K$ w.r.t. a basis of $H(X, k D)$ which is correct modulo $p^{N+1}$, where $N \geqslant 2+\left\lfloor\log _{p}(k+1)\right\rfloor$. Then the polynomial $\operatorname{det}\left(1-p^{-1} \tilde{A} T\right)$ as an approximation to $\operatorname{det}\left(1-T p^{-1} F \mid H_{\text {rig }}^{2}(U)\right)$ is correct modulo $p^{N+1-h^{2,0}-\left\lfloor\log _{p}(k+1)\right\rfloor}$.

This theorem is surprising since the matrix $p^{-1} \tilde{A}$ will not in general have entries in $W$, and so one naively expects a much greater loss of precision when the characteristic polynomial is computed. For example, when $p>k+1$ one expects naively that the characteristic polynomial of $p^{-1} \tilde{A}$ is only correct modulo $p^{N+1-(m-1)}$ where $m$ is the dimension of $H_{\text {rig }}^{2}(U)$. In our application to L-functions of elliptic curves, $m$ is much larger than $h^{2,0}$, and so Theorem 5.1 is of great practical use.

Theorem 5.1 is an immediate corollary of the next proposition.

Proposition 5.2. Assume that the residue field of $W$ is the prime field $\mathbb{F}_{p}$ where $p>2$, and denote by $h^{2,0}$ the geometric genus of $\mathcal{X}_{K}$ and by $g$ the genus of $\mathcal{D}_{K}$. Let $\mathcal{B}$ be a basis for the $W$-lattice $H(X, k D)$, and hence also the $K$-vector space $H(X, k D) \otimes_{W} K$. Let $A \in \mathrm{GL}(m, K)$ be the matrix for the Frobenius map $F$ acting on $H(X, k D) \otimes_{W} K$ w.r.t. this basis. Let $N \geqslant 2+\left\lfloor\log _{p}(k+1)\right\rfloor$ be a positive 
integer and $\tilde{A} \in \mathrm{GL}(m, \mathbb{Q})$ be a matrix such that $\operatorname{ord}_{p}(\tilde{A}-A) \geqslant N+1$. Write $\operatorname{det}(1-A T)=\sum_{\ell=0}^{m} a_{\ell} T^{\ell}$ and $\operatorname{det}(1-\tilde{A} T)=\sum_{\ell=0}^{m} \tilde{a}_{\ell} T^{\ell}$. Then

$$
\operatorname{ord}_{p}\left(a_{\ell}-\tilde{a}_{\ell}\right) \geqslant N+\ell+m(\ell)-\left\lfloor\log _{p}(k+1)\right\rfloor
$$

where

$$
m(\ell):= \begin{cases}-\ell+1 & 1 \leqslant \ell \leqslant h^{2,0} \\ -h^{2,0}+1 & h^{2,0}<\ell \leqslant h^{2,0}+h^{1,1}-1+g \\ -h^{2,0}+1+\left(\ell-\left(h^{2,0}+h^{1,1}-1+g\right)\right) & h^{2,0}+h^{1,1}-1+g<\ell \leqslant m .\end{cases}
$$

We shall prove this proposition in several simple steps. Using the notation and results in Section 4.2, we have an exact sequence (15)

$$
0 \rightarrow H(X)_{\text {prim }} \stackrel{\theta}{\rightarrow} H(X, \log D) \stackrel{\phi}{\rightarrow} H(D)(-1) \rightarrow 0
$$

which defines a mixed Frobenius-Hodge structure (Definition 3.12 ) on $H(X, \log D)$ from the pure Frobenius-Hodge structures on the outer terms. Say that a basis for $H(X)_{\text {prim }}$ is adapted to the Hodge filtration if the first $h^{2,0}$ elements form a basis for $H_{2}$, and the first $h^{2,0}+h^{1,1}-1$ form a basis for $H_{1}$ (see (13) for the Hodge filtration on $\left.H(X)_{\text {prim }}\right)$. Likewise, say that a basis for $H(D)$ is adapted to the Hodge filtration if the first $g$ elements form a basis for $H_{1}^{\prime}$ (see (14) for the Hodge filtration on $H(D)$ ). Say that a basis for $H(X, \log D)$ is adapted to the Hodge filtrations if the first $h_{2}-1$ elements are $\theta$ applied to a basis of $H(X)_{\text {prim }}$ which is adapted to the Hodge filtration, and $p^{-1} \phi$ applied to the last $2 g$ elements gives a basis of $H(D)$ which is adapted to the Hodge filtration. Note that $m=\left(h_{2}-1\right)+2 g$ is the rank of $H(X, \log D)$.

The matrix $A$ for Frobenius $F$ on a basis of $H(X, \log D)$ which is adapted to the Hodge filtrations has the shape

$$
A=\left(\begin{array}{ccccc}
p^{2} A_{2} & p A_{1} & A_{0} & B_{1} & B_{2} \\
0 & 0 & 0 & p^{2} C_{1} & p C_{2}
\end{array}\right)
$$

where $A_{0}, A_{2} \in \mathrm{M}\left(h_{2}-1, h^{2,0}, W\right), A_{1} \in \mathrm{M}\left(h_{2}-1, h^{1,1}-1, W\right), C_{i} \in \mathrm{M}(2 g, g, W)$, $B_{i} \in \mathrm{M}\left(h_{2}-1, g, W\right)$ and the 0 s are zero matrices of the appropriate size. Here $M(\star, \star, W)$ are matrix spaces of the relevant sizes over $W$.

LEMMA 5.3. Assume that the residue field of $W$ is the prime field $\mathbb{F}_{p}$ where $p>2$. Let $\mathcal{B}$ be a basis for $H(X, \log D)$ which is adapted to the Hodge filtrations and $A$ the matrix for Frobenius $F$ on $H(X, \log D)$ w.r.t. the basis $\mathcal{B}$. Let $N \geqslant 2$ be a positive integer and $\tilde{A} \in \mathrm{GL}(m, \mathbb{Q})$ be a matrix such that $\operatorname{ord}_{p}(\tilde{A}-A) \geqslant N+1$. Write $\operatorname{det}(1-A T)=\sum_{\ell=0}^{m} a_{\ell} T^{\ell}$ and $\operatorname{det}(1-\tilde{A} T)=\sum_{\ell=0}^{m} \tilde{a}_{\ell} T^{\ell}$. Then

$$
\operatorname{ord}_{p}\left(a_{\ell}-\tilde{a}_{\ell}\right) \geqslant N+\ell+m(\ell)
$$

where $m(\ell)$ is defined in the statement of Proposition 5.2.

Before proving Lemma 5.3, we need a definition and lemma.

Definition 5.4. Let $M=\left(m_{i, j}\right)$ be an $m \times m$ matrix over a commutative ring. $A$ (partial) transversal of $M$ is a choice of subset $\left\{u_{1}, \ldots, u_{\ell}\right\} \subseteq\{1,2, \ldots, m\}$ and permutation $\tau \in S_{\ell}$. A transversal product of $M$ is a product $\operatorname{sign}(\tau) m_{u_{1}, u_{\tau(1)}} \cdots m_{u_{\ell}, u_{\tau(\ell)}}$ for some choice of (partial) transversal. We say that the product contains the entries $m_{u_{1}, u_{\tau(1)}}, \ldots, m_{u_{\ell}, u_{\tau(\ell)}}$ of $M$. 
LEMMA 5.5. Let $M$ be an $m \times m$ matrix over a commutative ring and $M=$ $\left(M_{i, j}\right)_{1 \leqslant i, j \leqslant 2}$ a block decomposition where the block $M_{1,1}$ is square of size $m^{\prime}$, and the block $M_{2,2}$ is square of size $m^{\prime \prime}:=m-m^{\prime}$. Then any transversal product of $M$ must contain an equal number of entries from $M_{1,2}$ and $M_{2,1}$.

Proof. We may restrict immediately to the case in which the transversal is determined by choosing an element from every row and column of $M$, by extracting a suitable sub-matrix. For such a transversal, if $s$ elements are chosen from $M_{1,2}$ then by considering columns one sees $m^{\prime \prime}-s$ are chosen from $M_{2,2}$, and by considering rows that $m^{\prime}-s$ are chosen from $M_{1,1}$. Hence $m-\left(s+\left(m^{\prime}-s\right)+\left(m^{\prime \prime}-s\right)\right)=s$ are chosen from $M_{2,1}$.

We now prove Lemma 5.3.

Proof. Write $p^{-1} A=\left(p^{-1} a_{i, j}\right)$ and $p^{-1} \tilde{A}=\left(p^{-1} \tilde{a}_{i, j}\right)$, so $\operatorname{ord}_{p}\left(p^{-1} \tilde{a}_{i, j}-p^{-1} a_{i, j}\right) \geqslant$ $N \geqslant 2$. From the explicit block-form of the matrix $A$ immediately before the statement of the lemma, one sees that the matrix $V=\left(v_{i, j}\right)$ below is such that $\operatorname{ord}_{p}\left(p^{-1} a_{i, j}\right), \operatorname{ord}_{p}\left(p^{-1} \tilde{a}_{i, j}\right) \geqslant v_{i, j}$. Define $V$ to be the matrix

$$
\left(\begin{array}{ccccccccccccccc}
1 & \cdots & 1 & 0 & \cdots & 0 & -1 & \cdots & -1 & -1 & \cdots & -1 & -1 & \cdots & -1 \\
\vdots & & \vdots & \vdots & & \vdots & \vdots & & \vdots & \vdots & & \vdots & \vdots & & \vdots \\
1 & \cdots & 1 & 0 & \cdots & 0 & -1 & \cdots & -1 & -1 & \cdots & -1 & -1 & \cdots & -1 \\
N & \cdots & N & N & \cdots & N & N & \cdots & N & 1 & \cdots & 1 & 0 & \cdots & 0 \\
\vdots & & \vdots & \vdots & & \vdots & \vdots & & \vdots & \vdots & & \vdots & \vdots & & \vdots \\
N & \cdots & N & N & \cdots & N & N & \cdots & N & 1 & \cdots & 1 & 0 & \cdots & 0
\end{array}\right) .
$$

Explicitly, the top left-hand $\left(h_{2}-1\right) \times h^{2,0}$ sub-matrix of $1 \mathrm{~s}$ in $V$ corresponds to lower bounds on the valuation of entries in the matrix $p A_{2}$, and so on. Let $1 \leqslant \ell \leqslant m$. The eth coefficient of $\operatorname{det}\left(1-T p^{-1} \tilde{A}\right)$ can be written explicitly as a sum of transversal products of the entries $p^{-1} \tilde{a}_{i, j}$ in $p^{-1} \tilde{A}$, cf. [19, Equation (36)]. Each transversal product is determined by a choice of indices $1 \leqslant u_{1}<\cdots<u_{\ell} \leqslant m$ and permutation $\tau \in S_{\ell}$; namely, this defines the term $\operatorname{sign}(\tau)\left(p^{-1} \tilde{a}_{u_{1}, u_{\tau(1)}}\right) \cdots\left(p^{-1} \tilde{a}_{u_{\ell}, u_{\tau(\ell)}}\right)$. According to [19, Lemma 9.5], the "output" precision

$$
\operatorname{ord}_{p}\left(\left(p^{-1} a_{u_{1}, u_{\tau(1)}}\right) \cdots\left(p^{-1} a_{u_{\ell}, u_{\tau(\ell)}}\right)-\left(p^{-1} \tilde{a}_{u_{1}, u_{\tau(1)}}\right) \cdots\left(p^{-1} \tilde{a}_{u_{\ell}, u_{\tau(\ell)}}\right)\right)
$$

when computing such a product is bounded below by $N+v\left(u_{1}, \ldots, u_{\ell} ; \tau\right)$ where

$$
v\left(u_{1}, \ldots, u_{\ell} ; \tau\right):=\sum_{i=1}^{\ell} v_{u_{i}, u_{\tau(i)}}-\min _{1 \leqslant i \leqslant \ell}\left\{v_{u_{i}, u_{\tau(i)}}\right\}
$$

measures the change in precision. So for each $\ell$, we must find the minimum value taken by the function $v\left(u_{1}, \ldots, u_{\ell} ; \tau\right)$ over different choices of indices and permutation. By inspection of the matrix $V$, one sees that the minimum value is given by the function $m(\ell)$ defined in the statement of Proposition 5.2.

The key point is that since $N \geqslant 2$, one can restrict to transversal products of $p^{-1} A$ which factor as a transversal product of the $\left(h_{2}-1\right) \times\left(h_{2}-1\right)$ sub-matrix $\left(p A_{2}, A_{1}, p^{-1} A_{0}\right)$ times one of the $2 g \times 2 g$ sub-matrix $\left(p C_{1}, C_{2}\right)$, and it is easy to compute the minimum value of $v(\cdot)$ over such transversals. To see why we can restrict in this way, suppose that $1 \leqslant u_{1}<\cdots<u_{\ell} \leqslant m$ is a choice of indices 
and $\tau \in S_{\ell}$ of permutation such that the corresponding transversal product of $p^{-1} A$ contains exactly $s \geqslant 1$ elements from the top-right $h_{2}-1 \times 2 g$ sub-matrix of $p^{-1} A$. Then by Lemma 5.5, the transversal must also contain exactly $s$ elements from the bottom-left $2 g \times h_{2}-1$ sub-matrix of $p^{-1} A$. Hence $v\left(u_{1}, \ldots, u_{\ell} ; \tau\right)$ equals $(N-1) s \geqslant s$ plus a choice of $\ell-s$ elements lying in distinct columns (and rows) of the top-left $h_{2}-1 \times h_{2}-1$ sub-matrix of $V$ and the bottom-right $2 g \times 2 g$ sub-matrix of $V$, plus one. Such a sum cannot be less than $m(\ell)$, and moreover the value $m(\ell)$ is attained for some choice of transversal.

LEMMA 5.6. The statement of Lemma 5.3 remains true for $\mathcal{B}$ chosen to be any basis of $H(X, \log D)$.

Proof. Let $\mathcal{B}$ be any basis for $H(X, \log D)$ and $A$ the matrix for Frobenius $F$ w.r.t. $\mathcal{B}$. Let $\mathcal{B}^{\prime}$ be a basis adapted to the Hodge filtrations and $A^{\prime}$ the matrix for Frobenius $F$ w.r.t. $\mathcal{B}^{\prime}$. Of course, the characteristic polynomials of $A$ and $A^{\prime}$ are the same. Let $\tilde{A} \in \mathrm{GL}(\mathbb{Q}, m)$ be such that $\operatorname{ord}_{p}(\tilde{A}-A) \geqslant N+1$. Let $C \in \mathrm{GL}(m, W)$ be the change of basis matrix from $\mathcal{B}$ to $\mathcal{B}^{\prime}$, so $A^{\prime}=C^{-1} A C$. Define $D:=C^{-1} \tilde{A} C$, so the characteristic polynomials of $D$ and $\tilde{A}$ are identical. We have $D-A^{\prime}=C^{-1}(\tilde{A}-A) C$ so $\operatorname{ord}_{p}\left(D-A^{\prime}\right) \geqslant N+1$. Hence the characteristic polynomials of $D$ and $A^{\prime}$ agree as claimed in Lemma 5.3. Thus so do those of $\tilde{A}$ and $A$.

Lemma 5.7. Proposition 5.2 is true for bases $\mathcal{B}$ of $H(X, k D)$ which satisfy the following property: There exists a basis $\mathcal{B}^{\prime}$ of $H(X, \log D)$ with the change of basis matrix from $\mathcal{B}$ to $\mathcal{B}^{\prime}$ a diagonal matrix $E=\operatorname{diag}\left(p^{n_{1}}, \ldots, p^{n_{m}}\right)$ with $n_{i} \leqslant\left\lfloor\log _{p}(k+\right.$ 1)」.

Note that such bases exist since we know that $H(X, k D) / H(X, \log D)$ is a finite abelian $p$-group killed by multiplication by $p^{\left\lfloor\log _{p}(k+1)\right\rfloor}$ (Theorem 4.10$)$.

Proof. Define $G:=E^{-1} \tilde{A} E$ and $H:=E^{-1} A E$. Then $H$ is the matrix for Frobenius $F$ w.r.t. a basis of the lattice $H(X, \log D)$. Moreover, $G-H=E^{-1}(\tilde{A}-A) E$ and so $\operatorname{ord}_{p}(G-H) \geqslant(N+1)-\left\lfloor\log _{p}(k+1)\right\rfloor$. The characteristic polynomials of $G$ and $H$ are the same as those of $\tilde{A}$ and $A$, respectively, that is $\sum_{i=0}^{m} \tilde{a}_{i} T^{i}$ and $\sum_{i=0}^{m} a_{i} T^{i}$. By Lemma 5.6 (with notation " $A$ and $\tilde{A}$ " replaced by " $H$ and $G$ ", and $N$ replaced by $\left.N-\left\lfloor\log _{p}(k+1)\right\rfloor\right)$ they agree as claimed.

One now deduces Proposition 5.2 from Lemma 5.7 using a similar argument as that in the proof of Lemma 5.6.

\section{The inclusions $U \subset X$ and $V \subset U$}

In Section 6.2 we analyse the kernel and cokernel of the map $H_{r i g}^{n}(U) \rightarrow H_{r i g}^{n}(V)$ which arises in the fibration method. We restrict to the case in which the compactification $X$ of $U$ is a smooth surface in $\mathbb{P}_{\mathbb{F}_{q}}^{3}$ (Proposition 6.1) or a weighted projective surface subject to certain conditions (Proposition 6.2). The actual practical computation of this map does not cause any problems. Since both $U$ and $V$ are affine, cohomology classes can be represented by $n$-forms defined globally, and the map is just restriction. 
In Section 6.3 we briefly consider the problem of computing the residue map Res in the exact sequence

$$
\cdots \rightarrow H_{c r i s}^{n}(X) \rightarrow H_{c r i s}^{n}((X, D)) \stackrel{\text { Res }}{\rightarrow} H_{c r i s}^{n-1}(D)(-1) \rightarrow \cdots
$$

Given a basis for the full rank $W$-lattice $H(X, \log D)=\operatorname{Im}\left(H_{c r i s}^{n}((X, D)) \rightarrow\right.$ $\left.H_{\text {rig }}^{n}(U)\right)$, the sublattice $H(X)_{\text {prim }} \subseteq H(X, \log D)$ can in principle be recovered through an understanding of the residue map (Step 2 in Section 2.3.3).

\subsection{Excision exact sequences}

In this section we gather some exact sequences in rigid cohomology. Sequence (17) will be used in Section 6.2.

For $Y$ a smooth variety over $\mathbb{F}_{q}$ of pure dimension $d$ and $Z \subset Y$ a closed subscheme we have the localisation sequence [17, Section 4.4]

$$
\cdots \rightarrow H_{Z, \text { rig }}^{i}(Y) \rightarrow H_{\text {rig }}^{i}(Y) \rightarrow H_{\text {rig }}^{i}(Y \backslash Z) \rightarrow \cdots
$$

Here $H_{Z \text {,rig }}^{i}(Y)$ is rigid cohomology of $Y$ with support along $Z$. When $Z$ is smooth and pure of codimension $e$, one has the Gysin isomorphism [28]

$$
H_{\text {rig, },}^{i}(Y) \cong H_{\text {rig }}^{i-2 e}(Z)(-e)
$$

which leads to the more useful sequence

$$
\cdots \rightarrow H_{r i g}^{i-2 e}(Z)(-e) \rightarrow H_{r i g}^{i}(Y) \rightarrow H_{\text {rig }}^{i}(Y \backslash Z) \rightarrow \cdots
$$

Sequences (1) and (17) are related in so much as if one tensors the first by $K$ over $W$ one obtains a special case of the second. We only need (17) for our application in the next section. Finally, we recall that when $Y$ is affine $H_{\text {rig }}^{i}(Y)=0$ for $i>\operatorname{dim}(Y)$.

\subsection{Injectivity of the map $H_{\text {rig }}^{2}(U) \rightarrow H_{\text {rig }}^{2}(V)$}

The propositions in this section makes rigorous the ideas in [19, Section 9.3.1]. First, we consider the case of $X$ a smooth surface in $\mathbb{P}_{\mathbb{F}_{q}}^{3}$.

Proposition 6.1. Notation and assumptions as in the first paragraph of Section 3.2. Furthermore, let $\mathcal{U} \rightarrow \mathbb{A}_{W}^{1}$ have irreducible fibres, and let $\mathcal{V} \subset \mathcal{U}$ be such that $\mathcal{Z}:=\mathcal{U} \backslash \mathcal{V}$ is pure of dimension 1 and the restriction $\mathcal{V} \rightarrow \mathcal{S} \subset \mathbb{A}_{W}^{1}$ is a smooth morphism. Denote by $V, U$ and $S$ the special fibres of $\mathcal{V}, \mathcal{U}$ and $\mathcal{S}$. Then the map $H_{\text {rig }}^{2}(U) \rightarrow H_{\text {rig }}^{2}(V)$ is an injection.

Proof. First, $H_{\text {rig }}^{1}(X)=H_{\text {rig }}^{3}(X)=0$ since $X$ is a smooth surface in $\mathbb{P}_{\mathbb{F}_{q}}^{3}$. Writing out the sequence $(17)$ in full for the pair $(X, D)$ one obtains

$$
\begin{aligned}
& 0 \rightarrow H_{\text {rig }}^{0}(X) \rightarrow H_{\text {rig }}^{0}(U) \rightarrow \quad \rightarrow \quad 0 \\
& \rightarrow \quad 0 \quad \rightarrow \quad H_{\text {rig }}^{1}(U) \rightarrow H_{\text {rig }}^{0}(D)(-1) \\
& \stackrel{c}{\rightarrow} H_{\text {rig }}^{2}(X) \rightarrow H_{\text {rig }}^{2}(U) \rightarrow H_{\text {rig }}^{1}(D)(-1) \\
& \rightarrow \quad 0 \quad \rightarrow \quad 0 \quad \rightarrow \quad H_{\text {rig }}^{2}(D)(-1) \\
& \rightarrow H_{\text {rig }}^{4}(X) \quad \rightarrow \quad 0 \quad \rightarrow \quad 00 .
\end{aligned}
$$

Note that $H_{\text {rig }}^{0}(X)=H_{\text {rig }}^{0}(D)=K$. The image of the map $c$ is the class of the hyperplane section $D$, and thus the map is injective. Hence $H_{\text {rig }}^{1}(U)=0$.

We have $V \subset U$ with $Z:=U \backslash V$ pure of codimension 1 . We do not assume that $Z$ is smooth, so cannot apply the sequence (17) directly to the pair $(U, Z)$. To get 
around this problem, let $P$ denote the reduced subscheme of $Z$ associated to the singular locus on $Z$ (a union of points), and $\check{Z}:=Z \backslash P, \check{U}:=U \backslash P$. First, $(U, P)$ is a smooth pair, with $P$ of codimension 2 in $U$. So (17) for this pair is

$$
\begin{aligned}
& 0 \rightarrow \quad 0 \quad \rightarrow \quad H_{\text {rig }}^{0}(U) \rightarrow H_{\text {rig }}^{0}(\check{U}) \\
& \rightarrow \quad 0 \quad \rightarrow \quad H_{\text {rig }}^{1}(U) \rightarrow H_{\text {rig }}^{1}(\check{U}) \\
& \rightarrow \quad 0 \quad \rightarrow \quad H_{\text {rig }}^{2}(U) \rightarrow H_{\text {rig }}^{2}(\check{U}) \\
& \begin{array}{cccccc}
\rightarrow & 0 & \rightarrow & 0 & \rightarrow & H_{\text {rig }}^{3}(\check{U}) \\
\rightarrow & H_{\text {rig }}^{0}(P)(-2) & \rightarrow & 0 & \rightarrow & 0 .
\end{array}
\end{aligned}
$$

Note that $\check{U}$ is not affine when $P \neq \emptyset$. Second, $(\check{U}, \check{Z})$ is a smooth pair with $\check{Z}$ of codimension 1 in $\check{U}$ and $V=\check{U} \backslash \check{Z}$. So (17) for this pair is

$$
\begin{aligned}
& 0 \rightarrow \quad 0 \quad \rightarrow \quad H_{\text {rig }}^{0}(\check{U}) \rightarrow H_{\text {rig }}^{0}(V) \\
& \rightarrow \quad 0 \quad \rightarrow \quad H_{\text {rig }}^{1}(\check{U}) \rightarrow H_{\text {rig }}^{1}(V) \\
& \rightarrow H_{\text {rig }}^{0}(\check{Z})(-1) \rightarrow H_{\text {rig }}^{2}(\check{U}) \rightarrow H_{\text {rig }}^{2}(V) \\
& \rightarrow H_{\text {rig }}^{1}(\check{Z})(-1) \rightarrow H_{\text {rig }}^{3}(\check{U}) \rightarrow 0 .
\end{aligned}
$$

Thus the following sequence is exact

$$
\begin{gathered}
0 \rightarrow H_{\text {rig }}^{1}(V) \rightarrow H_{\text {rig }}^{0}(\check{Z})(-1) \rightarrow H_{\text {rig }}^{2}(U) \rightarrow H_{\text {rig }}^{2}(V) \\
\rightarrow H_{\text {rig }}^{1}(\check{Z})(-1) \rightarrow H_{\text {rig }}^{0}(P)(-2) \rightarrow 0 .
\end{gathered}
$$

To calculate $H_{\text {rig }}^{1}(V)$, we use the assumption that there is a smooth morphism $\mathcal{V} \rightarrow \mathcal{S} \subset \mathbb{A}_{W}^{1}$. Let $H_{r i g}^{i}(V / S)$ denote relative rigid cohomology for the special fibres of this map, where $i=0,1,2$. Then

$$
H_{\text {rig }}^{1}(V) \cong E_{2, \text { rig }}^{0,1} \oplus E_{2, \text { rig }}^{1,0},
$$

where

$$
\begin{aligned}
& E_{2, \text { rig }}^{1,0}:=\operatorname{Coker}\left(\frac{d}{d y}: H_{\text {rig }}^{0}(V / S) \rightarrow H_{\text {rig }}^{0}(V / S) \otimes \Omega_{A^{\dagger}}^{1}\right), \\
& E_{2, \text { rig }}^{0,1}:=\operatorname{Ker}\left(\frac{d}{d y}: H_{\text {rig }}^{1}(V / S) \rightarrow H_{\text {rig }}^{1}(V / S) \otimes \Omega_{A^{\dagger}}^{1}\right),
\end{aligned}
$$

with $y$ a coordinate function on the generic fibre $\mathcal{S}_{K}$ of the base $\mathcal{S}$, and the ring $A^{\dagger}$ the weak completion of $\Gamma\left(\mathcal{S}_{K}, \mathcal{O}_{\mathcal{S}_{K}}\right)$, cf. Section 8.2.1. Now $H_{\text {rig }}^{0}(V / S) \cong A^{\dagger}$ and so $E_{2, \text { rig }}^{1,0} \cong H_{\text {rig }}^{1}(S)$, and since the fibres of $U \rightarrow \mathbb{A}_{\mathbb{F}_{q}}^{1}$ are irreducible, the fibres of $\check{Z} \rightarrow \mathbb{A}_{\mathbb{F}_{q}}^{1} \backslash S$ are connected, and thus $H_{\text {rig }}^{1}(S) \cong H_{\text {rig }}^{0}(\check{Z})(-1)$, cf. [19, Proposition 8.1]. Thus (18) begins

$$
0 \rightarrow E_{2, \text { rig }}^{0,1} \oplus H_{\text {rig }}^{0}(\check{Z})(-1) \rightarrow H_{\text {rig }}^{0}(\check{Z})(-1) \rightarrow H_{\text {rig }}^{2}(U) \rightarrow \cdots
$$

and we deduce that $E_{2, \text { rig }}^{0,1}=0$ and $H_{\text {rig }}^{2}(U) \hookrightarrow H_{\text {rig }}^{2}(V)$.

Our conclusion is that the following sequence is exact

$$
0 \rightarrow H_{\text {rig }}^{2}(U) \rightarrow H_{\text {rig }}^{2}(V) \rightarrow H_{\text {rig }}^{1}(\check{Z})(-1) \rightarrow H_{\text {rig }}^{0}(P)(-2) \rightarrow 0
$$

and in particular the map $H_{\text {rig }}^{2}(U) \hookrightarrow H_{\text {rig }}^{2}(V)$ is an injection.

Next, we consider the case which arises in our application; that of a quasi-smooth hypersurface in a weighted projective space. 
Proposition 6.2. Notation and assumptions as in the first paragraph of Section 4. Furthermore, let $\mathcal{U} \rightarrow \mathbb{A}_{W}^{1}$ have irreducible fibres, and let $\mathcal{V} \subset \mathcal{U}$ be such that $\mathcal{Z}:=\mathcal{U} \backslash \mathcal{V}$ is pure of dimension 1 and the restriction $\mathcal{V} \rightarrow \mathcal{S} \subset \mathbb{A}_{W}^{1}$ is a smooth morphism. Let $V$ denote the special fibre of $\mathcal{V}$. Then the map $H_{\text {rig }}^{2}(U) \rightarrow H_{\text {rig }}^{2}(V)$ is an injection.

Proof. The proof is as before, once one has established that $H_{\text {rig }}^{1}(U)=0$. Now $H_{\text {rig }}^{1}(\tilde{X})=0$ since $\tilde{X}$ is a smooth hypersurface in $\mathbb{P}_{\mathbb{F}_{q}}^{3}$. The sequence $(17)$ for the pair $(\tilde{X}, \tilde{D})$ reveals that $H_{\text {rig }}^{1}(\tilde{U})=0$. Hence $H_{\text {rig }}^{1}(U)=H_{\text {rig }}^{1}(\tilde{U})^{G}=0$ (Lemma $4.5)$, as required.

\subsection{Computing the residue map}

In principle the residue map can be computed by using explicit Čech-de Rham complexes, following the approach of Gerkmann [11]. In unpublished notes, the author refined Gerkmann's work to obtain an explicit formula for the residue map in the case in which $\mathcal{X}:=\mathbb{P}_{W}^{2}$ and $\mathcal{D}$ is a curve on $\mathcal{X}$. Extending this to the case $\mathcal{X}:=\mathbb{P}_{W}^{3}$ and $\mathcal{D}$ is a surface proved too challenging for the present author, although plausible explicit expressions were obtained after much work. Note that the author carried out these latter calculations in an attempt to recover the lattice $\operatorname{Im}\left(H_{\text {cris }}^{2}(D) \rightarrow H_{\text {rig }}^{2}(D)\right)$ from the construction in [1] of the lattice $\operatorname{Im}\left(H_{\text {cris }}^{3}((X, D)) \rightarrow H_{\text {rig }}^{3}(X \backslash D)\right)$ via the residue map.

The author's experience with these calculations suggest that the explicit construction of the residue map in the case $\mathcal{X}$ is a surface and $\mathcal{D}$ is a smooth divisor is in principle possible, but perhaps not desirable in practice unless $H_{r i g}^{2}(U)$ has much larger dimension than $\operatorname{Im}\left(H_{\text {rig }}^{2}(X) \rightarrow H_{\text {rig }}^{2}(U)\right)$. In our calculations in Section 9 the former had dimension only two greater than the latter.

\section{The Fuchsian basis problem}

This section is entirely expository. We introduce the Fuchsian basis problem, and explain its relevance to our algorithm.

\subsection{Hilbert's 21st problem and Fuchsian bases}

Let $F$ be a field of characteristic zero and consider a linear differential system

$$
\frac{d C(y)}{d y}+B(y) C(y)=0,
$$

where $B(y)$ is an $m \times m$ matrix with entries in the rational function field $F(y)$, and the solution matrix $C(y)$ is an invertible matrix over some differential extension field of $F(y)$. If $H(y)$ is any invertible matrix of rational functions, we may transform (19) into an equivalent differential system in which $B$ is replaced by

$$
B_{[H]}:=H^{-1} B H+H^{-1} \frac{d H}{d y} .
$$

For an irreducible polynomial $p(y) \in F[y]$ let $F(y)_{p}:=F[y][1 / p]$ and $F(y)_{(p)}$ denote the local ring at $p$, i.e., the extension of $F[y]$ in which all irreducible polynomials excluding $p(y)$ have been inverted. We shall say that (19) is regular at $p(y) \in F[y]$ if there exists a matrix $H \in G L\left(m, F(y)_{p}\right)$ such that $B_{[H]}$ has entries in $F(y)_{(p)}$. We 
shall say that (19) is regular singular at $p(y) \in F[y]$ if it is not regular at $p(y)$ but there exists a matrix $H \in G L\left(m, F(y)_{p}\right)$ such that $p(y) B_{[H]}$ has entries in $F(y)_{(p)}$. We say that the system is regular/regular singular at infinity, if is regular/regular singular at $y$, after replacing the matrix $B(y)$ by $-1 / y^{2} B(1 / y)$. We call (19) regular singular if it is either regular or regular singular at all irreducible polynomials $p(y)$ and infinity.

For simplicity of exposition, let us henceforth assume that the system (19) is regular singular at infinity, and let $P(y)$ be the product of all irreducible polynomials $p(y)$ at which it is regular singular. Let us say $P(y)$ defines the finite singular locus. We shall say that (19) is Fuchsian if there exists a matrix $H \in G L(m, F[y, 1 / P(y)])$ such that $P(y) B_{[H]}$ has entries in $F[y]$ of degree less than $\operatorname{deg}(P(y))$. We call $B_{[H]}$ of this form a Fuchsian matrix for the differential system (19). Fuchsian systems are of course regular singular. Establishing whether the converse holds when $F$ is the field of complex numbers is closely related to Hilbert's 21st problem, though not the statement of it. We require a solution to the problem:

Fuchsian Basis Problem. Give an algorithm which takes as input a regular singular differential system (19) which is regular singular at infinity and has finite singular locus defined by the polynomial $P(y)$, and either

- gives as output a change of basis matrix $H(y) \in G L(m, F[y, 1 / P(y)])$ such that $B_{[H]}$ defined in (20) is a Fuchsian matrix for (19), or

- terminates and declares correctly that the system is not Fuchsian.

Hilbert's 21st problem has a chequered but fascinating history. When $F$ is the field of complex numbers, Fuchsian bases are known to exist for regular singular differential systems under various conditions, such as irreducibility. For $m=3$ there are examples of reducible systems which are regular singular but not Fuchsian; Bolibruch has made a very explicit study of this case [3]. For $m=2$, all regular singular systems over algebraically closed fields are Fuchsian, and an effective algorithm exists for finding Fuchsian bases (Dekkers's algorithm [6]).

\section{2. $\quad$ Picard-Fuchs systems}

Let $\mathcal{V}_{K} \rightarrow \mathcal{S}_{K} \subset \mathbb{P}_{K}^{1}$ be a smooth family of $K$-varieties of relative dimension $n-1$ over an affine base $\mathcal{S}_{K}$. Associated to this family is a free module $H_{d R}^{n-1}\left(\mathcal{V}_{K} / \mathcal{S}_{K}\right)$ of finite rank $m$, say, over the coordinate $\operatorname{ring} A:=\Gamma\left(\mathcal{S}_{K}, \mathcal{O}_{\mathcal{S}_{K}}\right)$, and an additive and Leibniz linear map (Gauss-Manin connection)

$$
\nabla: H_{d R}^{n-1}\left(\mathcal{V}_{K} / \mathcal{S}_{K}\right) \rightarrow H_{d R}^{n-1}\left(\mathcal{V}_{K} / \mathcal{S}_{K}\right) \otimes_{A} \Omega_{A}^{1}
$$

Choose a coordinate function $y$ on $\mathcal{S}_{K}$, and basis $e_{1}, \ldots, e_{m}$ for $H_{d R}^{n-1}\left(\mathcal{V}_{K} / \mathcal{S}_{K}\right)$. Then with respect to the choice of bases $\left\{e_{i}\right\}$ and $\left\{e_{i} \otimes d y\right\}$ for the domain and codomain of $\nabla$, the map acts as

$$
\frac{d}{d y}+B(y): A^{m} \rightarrow A^{m}
$$

for some matrix $B(y)$ with entries in $A$. The differential system associated to the Gauss-Manin connection and choice of initial basis $\left\{e_{i}\right\}$ and coordinate $y$ is defined to be (19). This system is regular singular, and has rational local exponents, by the regularity and local monodromy theorems $[\mathbf{1 3}]$. The method developed in $[\mathbf{1 9}$, Section 4] for computing in the cokernel of $\nabla$, and controlling the loss of $p$-adic 
precision which accrues, assumes that a basis has been chosen such that the matrix $B(y)$ for the Gauss-Manin connection is Fuchsian.

\section{Hyperelliptic curves over the rational function field}

In this section we begin by tying up various ends which were left loose in [19]. First, in that paper the author made a conjecture, the truth of which would improve the practical performance of the fibration method for hyperelliptic curves. This conjecture is proved in Section 8.2, at least under an assumption which holds true generically. Second, the algorithm assumed that certain local monodromy assumptions held true [19, Section 7.4]. In practice, it is easy to check computationally whether these assumptions hold, and they always do. We prove that these assumptions are true for the elliptic curves we consider in Section 9 by an explicit calculation (Section 8.3).

In Section 8.4 we make some informal comments on the complexity of the fibration method, incorporating the various refinements in this paper. Finally, in Section 8.5 we summarise the various conditions required by our refined fibration method to ensure the successful computation of L-functions of elliptic curves.

\subsection{Notation}

Let us first introduce the notation which we shall use in this section. Let $q$ be a power of an odd prime $p$, and $\bar{Q}(x, y) \in \mathbb{F}_{q}[x, y]$ have degree $2 g+1$ in the variable $x$ for some integer $g \geqslant 1$. Denote by $\bar{\Delta}(y)$ the Sylvester resultant of the polynomials $\bar{Q}$ and $\partial \bar{Q} / \partial x$ with respect to the variable $x$. We assume $\bar{\Delta}(y)$ is nonconstant. Let $Q(x, y) \in W[x, y]$ be some lift of the polynomial $\bar{Q}(x, y)$, and $\Delta$ the Sylvester resultant of $Q$ and $\partial Q / \partial x$ w.r.t. $x$. We need to choose $Q(x, y)$ so that $\operatorname{deg}(\Delta)=\operatorname{deg}(\bar{\Delta})>0$. This can certainly be done under some hypotheses, which are stated in Theorems 8.1, 8.2 and 8.4 with appropriate lifts taken in their proofs. Let $\mathcal{V} \rightarrow \mathcal{S}$ be the family obtained by taking "Spec" of the homomorphism

$$
W[y, 1 / \Delta(y)] \hookrightarrow W[x, y, 1 / \Delta(y), z] /\left(z^{2}-Q(x, y)\right) .
$$

Denote by $V \rightarrow S$ and $\mathcal{V}_{K} \rightarrow \mathcal{S}_{K}$ the (smooth) morphisms obtained by taking special and generic fibres of $\mathcal{V} \rightarrow \mathcal{S}$.

\subsection{A conjecture on the relative Frobenius matrix}

In this section we prove [19, Conjecture 7.4], under an assumption which holds true generically. This gives a useful practical improvement to the performance of the fibration method for hyperelliptic curves over function fields $\mathbb{F}_{q}(y)$.

\subsubsection{Statement of Conjecture 7.4}

We recall some notions from $[\mathbf{1 9}$, Section 7$]$, alerting the reader again to the fact that our notation has changed. Define $A^{\dagger}$ to be the weak completion of the coordinate ring of $\mathcal{S}_{K}$. That is

$$
A^{\dagger}:=\left\{\sum_{i=-\infty}^{\infty} \frac{a_{i}(y)}{\Delta(y)^{i}}\left|\operatorname{deg}\left(a_{i}\right)<\operatorname{deg}(\Delta), \operatorname{ord}_{p}\left(a_{i}\right)-\varepsilon\right| i \mid \rightarrow \infty \text { for some } \varepsilon>0\right\} .
$$

There is a monomorphism $\sigma_{A^{\dagger}}$ of $A^{\dagger}$ which lifts the $p$ th power map on $\mathbb{F}_{q}[y, 1 / \bar{\Delta}(y)]$ such that $\sigma_{A^{\dagger}}: y \mapsto y^{p}$. There is a rank $2 g$ free module $H_{\text {rig }}^{1}(V / S)$ over $A^{\dagger}$ with 
basis given by the classes of the forms $x^{i} d x / z$ for $i=0,1, \ldots, 2 g-1$. There is a map $F: H_{\text {rig }}^{1}(V / S) \rightarrow H_{\text {rig }}^{1}(V / S)$ which is $\sigma_{A^{\dagger}}$-linear, called the $p$ th power (relative) Frobenius map. We represent this map $F$ by a matrix $F(y)$ w.r.t. our choice of basis.

$[19$, Conjecture 7.4] The matrix $F(y)$ has a pole of finite order at $y=\infty$.

\subsubsection{Elliptic curves}

Assume that $\bar{Q}(x, y):=x^{3}+\bar{a}(y) x+\bar{b}(y)$ for some $\bar{a}(y), \bar{b}(y) \in \mathbb{F}_{q}[y]$. Then $\bar{\Delta}=$ $4 \bar{a}^{3}+27 \bar{b}^{2}$. We first consider a special case.

Theorem 8.1. Assume that $\operatorname{deg}(\bar{a}) \leqslant 4 e$ and $\operatorname{deg}(\bar{b}) \leqslant 6 e$ for some $e \geqslant 1$, and $\operatorname{deg}(\bar{\Delta})=12 e$. Then the entries in matrix $F(y)$ when expanded locally as Laurent series in $K((1 / y))$ have degree in $y$ at most $(p+1) e$; that is, the pole order of $F(y)$ at $y=\infty$ is bounded by $(p+1)$ e.

Proof. First, lift the elliptic curve equation to get $z^{2}=x^{3}+a(y) x+b(y)$ where $a, b \in W[y] \subset K(y)$ with $\operatorname{deg}(a) \leqslant 4 e, \operatorname{deg}(b) \leqslant 6 e$ and $\Delta:=4 a^{3}+27 b^{2}$ such that $\operatorname{deg}(\Delta)=12 e$. Note that any lifting satisfying the degree restrictions on $a$ and $b$ will be suitable. We make the change of variables $v:=1 / y, u:=v^{2 e} x$ and $w:=v^{3 e} z$ to get an equation $w^{2}=u^{3}+\tilde{a}(v) u+\tilde{b}(v)$. Here $\tilde{a}(v):=v^{4 e} a(1 / v) \in W[v]$ and $\tilde{b}(v):=v^{6 e} b(1 / v) \in W[v]$. The condition on the degree of the discriminant ensures us that $\tilde{\Delta}(v):=4 \tilde{a}^{3}+27 \tilde{b}^{2}=v^{12 e} \Delta(1 / v)$ does not vanish at $v=0$, even modulo $p$. So the lifted curve over $W[v]$ has good reduction at $v=0$, even modulo $p$. Recall that $d$ is the universal derivation of the function field of the generic fibre of the lifted curve over the base field $K(y)=K(v)$. We see that

$$
\frac{d x}{z}=v^{e} \frac{d u}{w}, \frac{x d x}{z}=v^{-e} \frac{u d u}{w} \text { and } \frac{d u}{w}=y^{e} \frac{d x}{z}, \frac{u d u}{w}=y^{-e} \frac{x d x}{z} .
$$

The matrix $F(y)$ expresses the action of the $p$ th power Frobenius map on the basis $d x / z$ and $x d x / z$. Let $F_{i, j}(y)$ be the $(i, j)$ th entry in $F(y)$, for $1 \leqslant i, j \leqslant 2$. These are $p$-adic functions which converge on some open annulus of outer radius 1 around $y=\infty$. We wish to show that they only have a finite polynomial part in $y$; thus they converge on the punctured open unit disk around $y=\infty$. Letting $\sigma$ denote the Frobenius action on functions and differentials we have

$$
\sigma\left(\frac{d x}{z}\right)=\sigma\left(v^{e} \frac{d u}{w}\right)=v^{e p} \sigma\left(\frac{d u}{w}\right) .
$$

The expression $\sigma\left(\frac{d u}{w}\right)$ may be computed using Kedlaya's method [19, Section 7.3.3], and written modulo exact differentials as a linear combination

$$
\sigma\left(\frac{d u}{w}\right) \equiv A_{1,1}(v) \frac{d u}{w}+A_{1,2}(v) \frac{u d u}{w} .
$$

The key point is that $A_{1,1}(v), A_{1,2}(v) \in K[[v]]$; thus these functions do not have poles at $v=0$. The reason for this is that since the lifted curve modulo $p$ is smooth at $v=0$, Kedlaya's reduction formulae do not introduce poles at this point. (Kedlaya's explicit method only introduces poles at singular fibres.) Similarly, we see that

$$
\sigma\left(\frac{x d x}{z}\right)=v^{-e p} \sigma\left(\frac{u d u}{w}\right) \equiv v^{-e p}\left(A_{2,1}(v) \frac{d u}{w}+A_{2,2}(v) \frac{u d u}{w}\right)
$$


where $A_{2,1}(v), A_{2,2}(v) \in K[[v]]$. Hence using (21) to get back to our original basis we find

$$
\begin{array}{ll}
F_{1,1}=y^{-e p} A_{1,1}(1 / y) y^{e}, & F_{1,2}=y^{-e p} A_{1,2}(1 / y) y^{-e}, \\
F_{2,1}=y^{e p} A_{1,2}(1 / y) y^{e}, & F_{2,2}=y^{e p} A_{2,2}(1 / y) y^{-e}
\end{array}
$$

where $A_{i, j} \in K[[1 / y]]$. Thus the matrix $F(y)$ has no positive powers of $y$ in the $(1,1)$ and $(1,2)$ positions, degree in $y$ bounded by $(p+1) e$ in the $(2,1)$ position, and degree bounded by $(p-1) e$ in the $(2,2)$ position.

The bounds in the theorem above on the pole order for the elliptic curves considered in Section 9 are $(p+1) e$ where $p=7$ and $e \in\{1,2,3,4,5\}$, that is $8,16,24,32,40$, and the actual pole orders observed were $6,12,18,24,30$, respectively.

We now consider a more general case, in which we impose only a generically held condition.

TheOREM 8.2. Assume $\operatorname{deg}(\bar{a}) \leqslant d / 3$ and $\operatorname{deg}(\bar{b}) \leqslant d / 2$ where the discriminant $\bar{\Delta}:=4 \bar{a}^{3}+27 \bar{b}^{2}$ has degree $d \geqslant 1$. Then the entries in matrix $F(y)$ when expanded locally as Laurent series in $K((1 / y))$ have degree in $y$ at most $\lfloor(p+1) d / 12\rfloor$; that is, the pole order of $F(y)$ at $y=\infty$ is bounded by $\lfloor(p+1) d / 12\rfloor$.

Proof. Lift the curve as before, preserving the degree of the discriminant. Let $m$ be the smallest integer such that $12 \mid m d$ where $d=\operatorname{deg}(\Delta(y)))$. Define $\tilde{y}:=y^{1 / m}$ and consider the generic fibre of the original lifted curve as being defined by the equation $z^{2}=x^{3}+a\left(\tilde{y}^{m}\right) x+b\left(\tilde{y}^{m}\right)$ over the totally ramified extension $K(\tilde{y}) / K(y)$. Then $\operatorname{deg}_{\tilde{y}}\left(\Delta\left(\tilde{y}^{m}\right)\right)=12 e$ for some integer $e \geqslant 1$. Now proceed as in the proof of Theorem 8.1, via the change of variables $v:=1 / \tilde{y}, u:=v^{2 e} x$ and $w:=v^{3 e} z$, to deduce that the Frobenius matrix $F(\tilde{y})$ has degree in $\tilde{y}$ bounded by $(p+1) e$. But this matrix converges on an open annulus around infinity, and so must have entries in $K((1 / y))$. Thus we deduce that the pole order at $y=\infty$ of $F(y)$ is bounded by $\lfloor(p+1) e / m\rfloor=\lfloor(p+1) d / 12\rfloor$.

Applying the above theorem to [19, Examples 9.1, 9.2], one obtains bounds on the pole order of $\lfloor(17+1) 26 / 12\rfloor=39$ and $\lfloor(5+1) 62 / 12\rfloor=31$, respectively, which are exactly the pole orders observed experimentally.

\subsubsection{Hyperelliptic curves}

The same technique can be used to prove $[\mathbf{1 9}$, Conjecture 7.4$]$ in greater generality; that is, first consider the case when the fibre $y=\infty$ is smooth, using an explicit change of basis, and then make a totally ramified extension of $K(y)$ to reduce to this case under a generically true assumption. We need the following lemma.

LEMMA 8.3. Let $g \geqslant 0$ and $\mathbb{Z}\left[a_{2}, a_{4}, \ldots, a_{2(2 g+1)}\right]$ be a graded polynomial ring, where $\operatorname{wt}\left(a_{i}\right):=i$. Then the discriminant of the polynomial $x^{2 g+1}+a_{2} x^{2 g}+a_{4} x^{2 g-1}+$ $\cdots+a_{4 g} x+a_{2(2 g+1)}$ is homogeneous of degree $2(2 g+1) 2 g$.

Proof. For $\lambda \in \mathbb{Q}^{*}$ the zero locus of the discriminant is invariant under the map $a_{2 i} \mapsto \lambda^{i} a_{2 i}(1 \leqslant i \leqslant 2 g+1)$, since on the original polynomial this transformation composed with $x \rightarrow \lambda x$ is just multiplication by $\lambda^{2 g+1}$. So the discriminant is weighted homogeneous w.r.t. the given weights. To compute the degree, specialise all but the constant coefficient to zero and use the explicit expression of the discriminant as the determinant of a Sylvester matrix. 
The next theorem subsumes Theorem 8.2.

TheOREM 8.4. Let $\bar{Q}(x, y) \in \mathbb{F}_{q}[x, y]$ be monic of degree $2 g+1$ in the variable $x$ for some integer $g \geqslant 1$. Let $\bar{\Delta}(y)$ be the discriminant of the hyperelliptic curve $z^{2}=\bar{Q}(x, y)$ over $\mathbb{F}_{q}(y)$, and $d:=\operatorname{deg}(\bar{\Delta})>0$. Assume that when one writes $\bar{Q}(x, y)=x^{2 g+1}+\bar{a}_{2}(y) x^{2 g}+\bar{a}_{4}(y) x^{2 g-1}+\cdots+\bar{a}_{2(2 g+1)}(y)$ we have

$$
\operatorname{deg}_{y}\left(\bar{a}_{i}(y)\right) \leqslant d \frac{i}{2(2 g+1) 2 g} .
$$

Then the entries in the matrix $F(y)$ when expanded locally as Laurent series in $K((1 / y))$ have degree in $y$ at most $\lfloor d(2 g-1)(p+1) /(4(2 g+1) g)\rfloor$; that is, the pole order of $F(y)$ at $y=\infty$ is bounded by $\lfloor d(2 g-1)(p+1) /(4(2 g+1) g)\rfloor$.

Proof. Lift the polynomial $\bar{Q}(x, y)$ to a polynomial $Q(x, y) \in W[x, y]$ preserving the discriminant degree. Lemma 8.3 and the assumptions on the degrees of the coefficients $\bar{a}_{i}(y)$ assures us that any lifting which preserves the degree bounds on the coefficients will be suitable. Let us first assume that $d=2(2 g+1) 2 g$ e for some integer $e$. Define $v:=1 / y, u:=v^{2 e} x$ and $w:=v^{(2 g+1) e} z$. Let $\tilde{Q}(u, v) \in K[u, v]$ be defined as $\tilde{Q}(u, v):=v^{d / 2 g} Q\left(v^{-2 e} u, 1 / v\right)$. Then the equation $w^{2}=\tilde{Q}(u, v)$ defines the generic fibre of the original lifted curve over $K(v)$ w.r.t. the new choice of variables. The discriminant $\tilde{\Delta}(v)$ of the polynomial $\tilde{Q}(u, v)$ equals $v^{d} \Delta(1 / v)$. Since we preserved the degree of the discriminant when lifting, $\tilde{\Delta}(0) \neq 0$, even modulo $p$, and the fibre at $v=0$ is smooth, even modulo $p$. We can compute the action of $F$ on the new basis $u^{i} d u / w$ for $0 \leqslant i<2 g$ using Kedlaya's algorithm. Let the $(i, j)$ th coefficients of the matrix thus obtained be $A_{i, j}(v)$. Since the fibre at $v=0$ is smooth, we have that the local expansions of the functions $A_{i, j}(v)$ at $v=0$ lie in $K[[v]]$.

Let $F_{i, j}(y)$ be the $(i, j)$ th coefficient of the matrix for the action of $F$ on the original basis $x^{i} d x / z, 0 \leqslant i<2 g$. Using the formulae

$$
\frac{x^{i} d x}{z}=v^{(2 g+1) e-2 e(i+1)} \frac{u^{i} d u}{w}, \frac{u^{j} d u}{w}=y^{(2 g+1) e-2 e(j+1)} \frac{x^{j} d x}{z}
$$

we see that

$$
\begin{aligned}
F_{i, j}(y) & =y^{-p[(2 g+1) e-2 e(i+1)]} y^{(2 g+1) e-2 e(j+1)} A_{i, j}(1 / y) \\
= & y^{2 e(p(i+1)-(j+1))-(p-1) e(2 g+1)} A_{i, j}(1 / y) .
\end{aligned}
$$

Thus the maximum degree in $y$ which occurs is bounded by

$$
2 e(2 g p-1)-(p-1) e(2 g+1)=e(2 g-1)(p+1) .
$$

The general case is treated by first making the change of variable $\tilde{y}:=y^{1 / m}$ where $m$ is the smallest integer such that $2(2 g+1) 2 g e=m d$ for some integer $e$. One deduces that the degree in $\tilde{y}$ of the matrix $F(\tilde{y})$ is bounded by $e(2 g-1)(p+1)$. Hence the degree of $F(y)$ in $y$ is bounded by $\lfloor e(2 g-1)(p+1) / m\rfloor=\lfloor d(2 g-1)(p+$ $1) /(4(2 g+1) g)\rfloor$.

Note that underlying the above proof is the fact that the generically held condition we insist upon relating the degrees of coefficients to that of the discriminant forces good or potentially good reduction at infinity. 
Applying the above theorem to [19, Example 9.3] one obtains a bound of $\lfloor 28(4-$ $1)(11+1) /(4(4+1) 2)\rfloor=25$ and the experimentally observed pole order is 21 .

\subsection{The Gauss-Manin connection for elliptic curves}

Write $\bar{Q}(x, y)=x^{3}+\bar{a}(y) x+\bar{b}(y)$ and $Q(x, y)=x^{3}+a(y) x+b(y)$, with other notation as in Section 8.1. Denote by $B(y)$ the matrix for the Gauss-Manin connection on $H_{d R}^{1}\left(\mathcal{V}_{K} / \mathcal{S}_{K}\right)$ with respect to the coordinate function $y$ and initial basis the cohomology classes of $d x / z$ and $x d x / z$, see Section 7.2 or [19, Section 7.3.1].

Proposition 8.5. The matrix for the Gauss-Manin connection with respect to our choice of basis, acting on the left on column vectors, is

$$
B(y)=\frac{1}{\Delta(y)}\left(\begin{array}{ll}
-a^{2} \frac{d a}{d y}-\frac{9}{2} b \frac{d b}{d y} & -a^{2} \frac{d b}{d y}+\frac{3}{2} a b \frac{d a}{d y} \\
-3 a \frac{d b}{d y}+\frac{9}{2} b \frac{d a}{d y} & a^{2} \frac{d a}{d y}+\frac{9}{2} b \frac{d b}{d y}
\end{array}\right) .
$$

Proof. An explicit calculation direct from the definition which uses the Sylvester matrix of $Q(x, y)=x^{3}+a(y) x+b(y)$ and $\frac{\partial Q}{\partial x}$ with respect to $x$, and Kedlaya's formula for pole reduction of differential forms $[\mathbf{1 6}]$.

Let $\bar{K}$ be an algebraic closure of $K$, and $R:=\{\gamma \in \bar{K} \mid \Delta(\gamma)=0\}$. Let $\mathrm{M}(K[y], 2)$ be the ring of $2 \times 2$ matrices over $K[y]$. Define $\beta(y) \in \mathrm{M}(K[y], 2)$ by $\beta(y):=$ $\Delta(y) B(y)$.

Lemma 8.6. The matrix $\beta(\gamma)$ is nilpotent for all $\gamma \in R$.

Proof. First, from Proposition 8.5 observe $\operatorname{Tr}(\beta(y))=0$. An explicit calculation reveals that

$$
\operatorname{det}(\beta(y))=-\frac{1}{4} \Delta(y)\left(a\left(\frac{d a}{d y}\right)^{2}+3\left(\frac{d b}{d y}\right)^{2}\right) .
$$

So at a root $\gamma$ of $\Delta(y)=0$ we have $\operatorname{det}(\beta(\gamma))=0$, and hence $\operatorname{det}(T-\beta(\gamma))=T^{2}$, as required.

Proposition 8.7. Assume that $\Delta(y)$ is squarefree, and let $\gamma \in R$. Let $t_{\gamma}:=y-\gamma$, a uniformizing parameter at $\gamma, K(\gamma)\left(\left(t_{\gamma}\right)\right)$ be the field of Laurent series, and embed $K[y, 1 / \Delta(y)] \hookrightarrow K(\gamma)\left(\left(t_{\gamma}\right)\right)$. Then

$$
B\left(t_{\gamma}\right)=r_{\gamma} t_{\gamma}^{-1}+s_{\gamma}\left(t_{\gamma}\right), s_{\gamma}\left(t_{\gamma}\right) \in \mathrm{M}\left(K(\gamma)\left[\left[t_{\gamma}\right]\right], 2\right)
$$

where the residue matrix $r_{\gamma} \in \mathrm{M}(K(\gamma), 2)$ is nilpotent.

Proof. We have

$$
B(y)=\frac{\beta(y)}{\Delta(y)}=\frac{\beta(y)}{\Delta^{\prime}(y)} \sum_{\gamma \in R} \frac{1}{y-\gamma} .
$$

Let $\gamma \in R$. Then $B\left(t_{\gamma}\right)$ can be written as claimed where $r_{\gamma}:=\beta(\gamma) / \Delta^{\prime}(\gamma)$. Since $\beta(\gamma)$ is nilpotent, it follows that $r_{\gamma}$ is nilpotent.

Thus under the hypothesis that $\Delta(y)$ is squarefree, the Gauss-Manin has regular singular points at the roots of $\Delta(y)=0$, with nilpotent residue matrix. So we have proved in an entirely explicit manner the following well-known result. 
COROLlary 8.8. When the discriminant $\Delta(y)$ is non-constant and squarefree, the family of elliptic curves defined by the equation $z^{2}=x^{3}+a(y) x+b(y)$ has regular singularities and unipotent local monodromy at the roots of $\Delta(y)=0$.

We now consider the local monodromy at infinity. First, let us consider the case which arises in our calculations in Section 9.

LEMmA 8.9. Assume that $\operatorname{deg}(a) \leqslant \operatorname{deg}(b) / 2$ and $\operatorname{deg}(\Delta)=2 \operatorname{deg}(b)$. Then we have $\operatorname{deg}_{y}(B(y)) \leqslant-1$, and the coefficient of $y^{-1}$ in the local expansion at zero of $B(y)$ has the form

$$
\frac{1}{27}\left(\begin{array}{cc}
-\frac{9}{2} \operatorname{deg}(b) & \star \\
0 & \frac{9}{2} \operatorname{deg}(b)
\end{array}\right)
$$

where $\star=0$ when $\operatorname{deg}(a)<\operatorname{deg}(b) / 2$. When $\operatorname{deg}(a)=\operatorname{deg}(b) / 2$ we have

$$
\star=\frac{\alpha^{2}}{\beta}\left(-\operatorname{deg}(b)+\frac{3}{2} \operatorname{deg}(a)\right)=-\frac{\alpha^{2}}{4 \beta} \operatorname{deg}(b),
$$

where $\alpha$ and $\beta$ are the leading coefficients of $a$ and $b$, respectively.

Proof. This can be read off from the connection matrix in Proposition 8.5.

When $\operatorname{deg}(b) \equiv 0 \bmod 6$, as in Section 9, the residue matrix has integer eigenvalues $\pm \operatorname{deg}(b) / 6$. Note that since the residue matrix is not prepared in this case, one cannot apply directly the precision loss bounds deduced in [19, Section 4] when reducing the "polynomial part" of differential forms. However, by virtue of our proof of [19, Conjecture 7.4], very little reduction needs to be done on the "polynomial part", and naive estimates are sufficient.

When $\operatorname{deg}(a) \geqslant \operatorname{deg}(b)$ and $\operatorname{deg}(\Delta)=3 \operatorname{deg}(a)$, the matrix $B(y)$ is such that $\operatorname{deg}_{y}(B(y)) \leqslant-1$, and one can explicitly read off the residue matrix. However, in the case $\operatorname{deg}(b) / 2<\operatorname{deg}(a)<\operatorname{deg}(b)$ the entry $B_{1,2}(y)$ does not have a simple pole at infinity and one needs to make a change of basis. We explain how to do this in the proof of Proposition 8.10, although we do not examine the local monodromy at infinity. Note that we have not implemented this change of basis routine in our algorithm as it requires an extension of the base field. The author does not know whether a Fuchsian basis exists for the general case without first extending the base field.

Assume $\operatorname{deg}(b) / 2<\operatorname{deg}(a)<\operatorname{deg}(b)$ and

$$
d:=\operatorname{deg}(\Delta)=\max \{2 \operatorname{deg}(b), 3 \operatorname{deg}(a)\}>0 .
$$

When $\operatorname{deg}(a) \leqslant 2 \operatorname{deg}(b) / 3$ define $m:=2 \operatorname{deg}(a)-\operatorname{deg}(b)$, and when $\operatorname{deg}(a)>$ $2 \operatorname{deg}(b) / 3$ define $m:=\operatorname{deg}(b)-\operatorname{deg}(a)$. Note that $m+1$ is the pole order at infinity of $B_{1,2}(y)$. Assume that $\Delta(y)$ is squarefree, and also that either $B_{1,1}(y)$ or $B_{2,1}(y)$ is non-zero.

Proposition 8.10. Let $L \supseteq K$ be such that $\Delta(y)$ has a root $\gamma \in L$ and a factor of degree $m$ defined over $L$. Then there exists a basis for $H_{d R}^{1}\left(\mathcal{V}_{K} / \mathcal{S}_{K}\right) \otimes_{K} L$ such that the matrix for the Gauss-Manin connection $\nabla$ is Fuchsian (see Section 7.1 for the definition of this term).

Proof. After a change of variable $y \mapsto y-\gamma$ we may assume $\gamma=0$. We make a further change of variable $t:=1 / y$. The matrix for the connection $\nabla$ with respect to 
the same basis but different choice of coordinate function $t$ is $\tilde{B}(t):=-1 / t^{2} B(1 / t)$. Define $\tilde{\Delta}:=t^{d} \Delta(1 / t)$. Then from Proposition 8.5 one sees

$$
\tilde{B}(t)=\left(\begin{array}{ll}
\frac{c(t)}{t \tilde{\Delta}(t)} & \frac{d(t)}{t^{m+1} \tilde{\Delta}(t)} \\
\frac{t^{m-1} e(t)}{\tilde{\Delta}(t)} & -\frac{c(t)}{t \tilde{\Delta}(t)}
\end{array}\right) \text {, where } c(t), d(t), e(t) \in L[t], e(t) \text { or } c(t) \text { non-zero. }
$$

Moreover, since $B(y)$ had a simple pole at $y=0$, one sees that $\operatorname{deg}(c) \leqslant \operatorname{deg}(\tilde{\Delta})$, $\operatorname{deg}(d) \leqslant \operatorname{deg}(\tilde{\Delta})+m$ and $\operatorname{deg}(e) \leqslant \operatorname{deg}(\tilde{\Delta})-m$. Now make a change of basis using the matrix

$$
H(t):=\left(\begin{array}{ll}
g(t) & \alpha(t) \\
0 & t^{m}
\end{array}\right)
$$

where $g(t)$ is a factor of $\tilde{\Delta}(t)$ of degree $m$, and $\alpha(t) \in L[t]$ will shortly be chosen. One computes that $\tilde{B}_{[H]}($ see $(20))$ equals

$$
\left(\begin{array}{ll}
\frac{c-\alpha e}{t \tilde{\Delta}}+\frac{g^{\prime}}{g} & \frac{2 c \alpha-e \alpha^{2}+d}{t \tilde{\Delta} g}+\frac{\alpha^{\prime}}{g}-\frac{m \alpha}{t g} \\
\frac{e g}{t \tilde{\Delta}} & \frac{e \alpha-c}{t \tilde{\Delta}}+\frac{m}{t}
\end{array}\right)
$$

Choose $\alpha \in K[t]$ with $\operatorname{deg}(\alpha)<\operatorname{deg}(g)=m$ such that

$$
-e \alpha^{2}+2 c \alpha+d=g f
$$

for some $f \in W[t]$; that is, solve the quadratic equation $-e X^{2}+2 c X+d=0 \bmod g$. This is always possible without extending the field further: the discriminant of the equation is $4 c^{2}+4 e d$, which is zero modulo $\tilde{\Delta}(t)$ since the residue matrices of $\tilde{B}(t)$ are nilpotent at the roots of $\tilde{\Delta}(t)$ (by Proposition 8.7). With this choice of $\alpha$ one sees that the entries in the matrix $\tilde{B}_{[H]}$ are of the form $\star / t \tilde{\Delta}(t)$ where $\operatorname{deg}(\star) \leqslant \operatorname{deg}(\tilde{\Delta})$.

\subsection{Complexity estimates}

All of the complexity estimates in this section are for deterministic algorithms. Let $\bar{Q}(x, y) \in \mathbb{F}_{q}[x, y]$ with $\operatorname{deg}_{x}(\bar{Q})=2 g+1$ and $h:=\operatorname{deg}_{y}(\bar{Q})$. From $[\mathbf{2 0}$, Theorem 1] we see that one may compute the L-function of a hyperelliptic curve $z^{2}=\bar{Q}(x, y)$ over $\mathbb{F}_{q}(y)$ in $(p g h \log (q))^{C}$ bit operations for some absolute constant $C \geqslant 1$. For $g$ fixed and $h / p$ bounded, [19, Theorem 8.6] tells us we may compute the L-function in $\mathcal{O}\left(p^{1+\varepsilon} \log (q)^{3+\varepsilon} h^{4+\varepsilon}\right)$ bit operations, for any $\varepsilon>0$ (under various hypotheses stated therein). Let us now assume $\bar{Q}(x, y)$ satisfies the hypothesis in the statement of Theorem 8.4. For $h$ fixed, using ideas similar to those in the last paragraph of $[\mathbf{1 9}$, Section 8.3] and Theorem 8.4 one can show that the L-function may be computed in $\mathcal{O}\left(p^{1+\varepsilon} \log (q)^{3+\varepsilon} g^{7+\varepsilon}\right)$ bit operations, for any $\varepsilon>0$ (under the same hypotheses). The main point is that the bound in Theorem 8.4 does not grow with $g$. This last bound does not use any of the refinements in this paper other than the proof of [19, Conjecture 7.4].

Assume now that $g$ is fixed. The difficulty lies in proving bounds when $h / p$ is allowed to grow. The problem is that using the method in [19], even combined with our proof of [19, Conjecture 7.4], one can only show that the matrix of the $p$ th power Frobenius on $H_{\text {rig }}^{2}(V)$ has valuation bounded below by some absolute constant times $-h / p$. This lower bounds impacts on the complexity when one computes the characteristic polynomial, because of the possibility of precision loss. 
Let us now assume that we are in the situation in the first paragraph of Section 4.3.1, and moreover the various hypotheses required in [19, Theorem 7.6] also hold true. By computing Frobenius on the basis elements of the lattice $H(X, k D)$ in Section 4.3 one gets a matrix whose valuation is bounded below by some absolute constant times $-\log _{p}(h)$, and so there is no problem with precision loss when one computes the characteristic polynomial. Unfortunately, as yet the author has not been able to prove useful bounds during another step of the refined algorithm; namely after reduction in the cokernel of $\nabla$, when an element of $H(X, k D) \otimes_{W} K$ written w.r.t. a given generating set for $H_{\text {rig }}^{2}(V)$ is written as a linear combination of the chosen basis for $H(X, k D)$. In practice, this does not cause any problems: this step can be accomplished by multiplication by an explicit matrix with entries in an algebraic number field, and one can measure the loss of precision simply by computing the valuation of the matrix at the prime $p$. In the examples computed in Section 9, this loss of precision was zero; note that this precision loss can be computed right at the start of the algorithm, before various precisions are chosen. Assuming the loss of precision at this step is bounded by some polynomial function of $\log _{p}(h)$, then one gets a complexity of $\mathcal{O}\left(p^{1+\varepsilon} \log (q)^{3+\varepsilon} h^{4+\varepsilon}\right)$ bit operations, for any $\varepsilon>0$.

\subsection{Comments for elliptic curves}

We conclude by making a summary of the conditions required for the application of our refined fibration algorithm (Section 2.3.3) to compute the L-function of an elliptic curve $z^{2}=x^{3}+\bar{a}(y) x+\bar{b}(y)$ over a field $\mathbb{F}_{q}(y)$ where $\bar{a}, \bar{b} \in \mathbb{F}_{q}[y]$ and $q$ is odd. Note that we omit Step 2, and only carry out Step 1 "approximately" — see the comments following the algorithm.

Taking the last step first, for Step 4 one needs that $\bar{\Delta}:=4 \bar{a}^{3}+27 \bar{b}^{2}$ is squarefree and $\operatorname{deg}(\bar{a}) \leqslant \operatorname{deg}(\bar{b}) / 2$ with $p>3$, so that the matrix for the Gauss-Manin connection is Fuchsian. (By Proposition 8.10 the degree restriction can be circumvented if one is willing to extend the base field.) Moreover, we need that the discriminant does not vanish at some point in $\mathbb{F}_{q}$, so there is a smooth fibre defined over the base field. For Steps 1 and 3 we need to assume that the conditions stated in the first paragraph of Section 4 hold true, and moreover that $\mathbb{F}_{q}$ is a prime field, since our analysis for Theorem 5.1 was only carried out for such fields. The essential assumption here is that the affine surface $\mathcal{U}$ defined by the equation $z^{2}=x^{3}+a(y) x+b(y)$ over $W$ is smooth, so that the rigid cohomology space $H_{\text {rig }}^{2}(U)$ is well-behaved; here $a$ and $b$ are liftings of $\bar{a}$ and $\bar{b}$ preserving the degree of the discriminant $\bar{\Delta}$. By $[\mathbf{2 4}$, Lemma IV.9.5.(a)], smoothness follows from the assumption that $\bar{\Delta}$ is squarefree. The construction of lattices in Section 4 requires a further smoothness hypothesis and coprimality condition. For the case in which the discriminant degree is $d=12 e$, one requires that $p$ does not divide $e$ and that the projective surface defined by the affine equation $z^{12 e}=x^{12 e}+a(y) x^{4 e}+b(y)$ is smooth. These assumptions are needed so that Theorem 5.1 may be applied; however, the author believes that this theorem should hold true under only the condition that $p$ does not divide $e$, cf. Note 4.12. Finally, we note that our implementation will fail if torsion is encountered during the construction of the lattice $H(X, k D)$ in Section 4.3.2 (this has never happened). Under all these conditions, our output is provably correct. 
Ranks of elliptic curves over function fields

\begin{tabular}{||l|ccccc||}
\hline & $d=6$ & $d=12$ & $d=18$ & $d=24$ & $d=30$ \\
\hline analytic rank 0 & 336 & 378 & 449 & 473 & 505 \\
\hline analytic rank 1 & 481 & 489 & 489 & 496 & 482 \\
\hline analytic rank 2 & 162 & 120 & 55 & 31 & 13 \\
\hline analytic rank 3 & 19 & 11 & 7 & 0 & 0 \\
\hline analytic rank 4 & 2 & 2 & 0 & 0 & 0 \\
\hline analytic rank >4 & 0 & 0 & 0 & 0 & 0 \\
\hline
\end{tabular}

Table 1: Analytic ranks of elliptic curves of conductor degree $2 d$

\section{Ranks of elliptic curves}

For each $d \in\{6,12,18,24,30\}$, the author selected in a verifiably pseudorandom manner 1000 elliptic curves of the form

$$
z^{2}=x^{3}+\bar{a}(y) x+\bar{b}(y)
$$

where $\bar{a}, \bar{b} \in \mathbb{F}_{7}[y]$ with $\operatorname{deg}(\bar{b})=d$ and $\operatorname{deg}(\bar{a}) \leqslant d / 2$, subject to the condition that the discriminant is squarefree and does not vanish at $y=0$. We imposed the further condition that the projective surface defined by the affine equation $z^{2 d}=x^{2 d}+\bar{a}(y) x^{2 d / 3}+\bar{b}(y)$ is smooth, cf. Note 9.1. The L-functions of the curves were computed using the refined fibration method. These are polynomials of degree $2 d-4$ with all reciprocal roots of complex absolute value 7 . Table 1 details the number of curves of each analytic rank. (The analytic rank of an elliptic curve over $\mathbb{F}_{q}(y)$ is the multiplicity of the integer $q$ as a reciprocal root of the L-function of that curve. This is an upper bound on the rank of the group of rational points on the curve over $\mathbb{F}_{q}(y)[\mathbf{1 4}$, Page 12], and the Tate conjecture asserts this upper bound is an equality $[\mathbf{2 6}, \mathbf{2 7}]$.)

Assuming the Tate conjecture, one observes that the percentage with infinitely many rational points is $66.4 \%, 62.2 \%, 55.1 \%, 52.7 \%$ and $49.5 \%$, respectively neatly flattening from the previous experimentally observed fraction of around two thirds to around the predicted value of one half. (The Tate conjecture is known in the cases $d=6$ and $d=12$, since these are rational surfaces and elliptic K3 surfaces $[27$, Theorems 5.2(b), 5.6(b)].)

Note 9.1. The additional generic condition that the projective surface defined by the affine equation $z^{2 d}=x^{2 d}+\bar{a}(y) x^{2 d / 3}+\bar{b}(y)$ is smooth is an artifact of the method of proof in Section 4, and undoubtedly irrelevant to the algorithm and the correctness of its output (though not proven to be so within this paper). Table 2 details analytic ranks for the first 1000 curves chosen without imposing this condition.

Assuming the Tate conjecture, one observes that the percentage with infinitely many rational points here is $65.4 \%, 62.1 \%, 54.7 \%, 52.1 \%$ and $50.3 \%$, respectively.

Note 9.2. The restriction that $\operatorname{deg}(\bar{a}) \leqslant d / 2$, rather than the more natural $\operatorname{deg}(\bar{a}) \leqslant 2 d / 3$, forces the $j$-invariants of the curves chosen to vanish to a high order at infinity. So we have not chosen curves randomly in a dense open subset of all elliptic curves with discriminant of degree $2 d$. However, the curves are chosen 
Ranks of elliptic curves over function fields

\begin{tabular}{||l|ccccc||}
\hline & $d=6$ & $d=12$ & $d=18$ & $d=24$ & $d=30$ \\
\hline analytic rank 0 & 346 & 379 & 453 & 479 & 497 \\
\hline analytic rank 1 & 471 & 494 & 493 & 493 & 493 \\
\hline analytic rank 2 & 162 & 114 & 47 & 28 & 10 \\
\hline analytic rank 3 & 20 & 11 & 7 & 0 & 0 \\
\hline analytic rank 4 & 1 & 2 & 0 & 0 & 0 \\
\hline analytic rank >4 & 0 & 0 & 0 & 0 & 0 \\
\hline
\end{tabular}

Table 2: Analytic ranks of elliptic curves chosen in Note 9.1

uniformly at random from a dense open subset of a "Weiestrass family" which for $d \geqslant 12$ has geometric monodromy group the full orthogonal group $O(2 d-4)[\mathbf{1 5}$, Theorem 10.2.13]. What we are really testing experimentally are the predictions of Katz for such families as one allows the degree of the conductor to grow $[\mathbf{1 4}$, Introduction]. These predictions include that asymptotically exactly one half will have infinitely many rational points.

The curves used in this section and their unitarised L-functions, along with the details of how the curves were generated, are presented as an electronic appendix to this paper. Precisely, each of the MAGma files "UnitarisedLfunctions-p7d $\star$.m" for $\star \in\{6,12,18,24,30\}$ contains 1250 elliptic curves and their unitarised L-functions. The Magma file "Random-Input.m" contains code which allows the reader to recover the pseudorandom input and text explaining how Tables 1 and 2 were derived from the data in "UnitarisedLfunctions-p7d $\mathrm{d} . \mathrm{m}$ ".

\section{References}

1. T.G. Аввот, K.S. Kedlaya and D. Roe, 'Bounding Picard numbers of surfaces using p-adic cohomology', Arithmetic, Geometry and Coding Theory (AGCT 2005), Société Mathématique de France, to appear. Available at arxiv:math/0601508v2. 177, 180, 182, 183, 199

2. B. Bektemirov, B. Mazur, W. Stein and M. Watkins, 'Average ranks of elliptic curves: tension between data and conjecture', Bull. Amer. Math. Soc. 44 (2007) 233-254. 172

3. A. A. Bolibrukh, 'The Riemann-Hilbert problem', Russian Math. Surveys 45(2) (1990) 1-47. 200

4. D. A. Cox and S. KATZ, Mirror symmetry and algebraic geometry, Mathematical Surveys and Monographs 68 (American Mathematical Society, Providence, RI, 1999). 190

5. D. Cox, J. Little and D. O'SheA, Ideals, varieties, and algorithms, Undergraduate Texts in Mathematics, 2nd edn (Springer, New York, 1997). 188

6. W. DEKKERS, 'The matrix of a connection having regular singularities on a vector bundle of rank 2 on $P^{1}(C)$ ', Lecture Notes in Mathematics 712 (Springer, Berlin, 1979) 33-43. 200 
7. P. Deligne, 'La conjecture de Weil: II', Publ. Inst. Hautes Études Sci. 52 (1981) 313-428. 176

8. A. DimcA, 'Singularities and coverings of weighted complete intersections', J. reine angew. Math. 366 (1986) 184-193. 187

9. A. DimcA, Singularities and topology of hypersurfaces (Springer, Berlin, 1992). 189

10. A. DimcA, Sheaves in topology (Springer, Berlin, 2004). 182, 183

11. R. GERKMAnn, 'Relative rigid cohomology and deformation of hypersurfaces', Int. Math. Res. Pap. 2007 (2007), article ID rpm003, 67 pages. 199

12. R. Hartshorne, Algebraic geometry, Graduate Texts in Mathematics 52 (Springer, New York, 1977). 183, 184

13. N. M. KATZ, 'Nilpotent connections and the monodromy theorem: applications of a result of Turrittin', Publ. Inst. Hautes Études Sci 39 (1970) 175-232. 200

14. N. M. KATZ, Twisted L-functions and monodromy, Annals of Mathematics Studies 150, (Princeton University Press, Princeton, NJ, 2002). 209, 210

15. N. M. KATZ, Moments, monodromy, and perversity: a diophantine perspective, Annals of Mathematics Studies 159 (Princeton University Press, Princeton, NJ, 2005). 210

16. K. S. KedlayA, 'Counting points on hyperelliptic curves using MonskyWashnitzer cohomology', J. Ramanujan Math. Soc. 16 (2001) 323-338. 205

17. K. S. Kedlaya, 'Finiteness of rigid cohomology with coefficients', Duke Math. J. 134 (2006) 15-97. 177, 197

18. K.S. KeDlayA, 'Search techniques for root-unitary polynomials', Proceedings of a Special Session on Computational Arithmetic Geometry (K. Lauter and K. Ribet, eds), American Mathematican Society, to appear. Available at arxiv:math/0611835v3. 174

19. A. G. B. LAUDER, 'A recursive method for computing zeta functions of varieties', LMS J. Comput. Math. 9 (2006) 222-267. 172, 173, 174, 175, 178, 195, 197, 198, 200, 201, 202, 203, 205, 206, 207, 208

20. A. G. B. LAUder and D. WAN, 'Counting points on varieties over finite fields of small characteristic', Algorithmic number theory: lattices, number fields, curves and cryptography (ed. J.P. Buhler and P. Stevenhagen), Math. Sci. Res. Inst. Publ. 44, to appear. Available at arxiv:math/0612147. 207

21. B. Le Stum, Rigid cohomology, Cambridge Tracts in Mathematics 172 (Cambridge University Press, Cambridge, 2007). 175

22. B. Mazur, 'Frobenius and the Hodge filtration', Bull. Amer. Math. Soc. 78 (1972) 653-667. 185, 186

23. J. S. Milne, Etale cohomology, Princeton Mathematical Series (Princeton University Press, Princeton, 1980). 179

24. J. H. Silverman, Advanced topics in the arithmetic of elliptic curves, Graduate Texts in Mathematics 151 (Springer, New York, 1994). 208

25. J. STEEnBRInk, 'Intersection form for quasi-homogeneous singularities', Compositio Math. 34 (1977) 211-223. 189, 190 
26. J. TAte, 'Algebraic cycles and poles of zeta functions', Arithmetic algebraic geometry, Proc. Conf. Purdue Univ., 1963 (Harper and Row, New York, 1965) 93-110. 172, 209

27. J. TAte, 'Conjectures on algebraic cycles in $\ell$-adic cohomology', Proc. Sympos. Pure Math. 55 (1994) Part 1, 71-83. 209

28. N. Tsuzuki, 'On the Gysin isomorphism in rigid cohomology', Hiroshima Math. J. 29 (1999) 479-527. 197

Alan G.B. Lauder lauder@maths.ox.ac.uk

Mathematical Institute 24-29 St Giles, Oxford OX1 3LB

United Kingdom 